ARTICLE

\title{
Structure of a Ty1 restriction factor reveals the molecular basis of transposition copy number control
}

\author{
Matthew A. Cottee (1) 1,7, Sean L. Beckwith (1) 2,7, Suzanne C. Letham (1) 1,6, Sarah J. Kim², George R. Young (1) 3,4, \\ Jonathan P. Stoye (10) ${ }^{4,5}$, David J. Garfinkel (1) ${ }^{2 \otimes} \&$ lan A. Taylor (D) ${ }^{1 凶}$
}

Excessive replication of Saccharomyces cerevisiae Ty1 retrotransposons is regulated by Copy Number Control, a process requiring the p22/p18 protein produced from a sub-genomic transcript initiated within Ty1 GAG. In retrotransposition, Gag performs the capsid functions required for replication and re-integration. To minimize genomic damage, p22/p18 interrupts virus-like particle function by interaction with Gag. Here, we present structural, biophysical and genetic analyses of p18m, a minimal fragment of Gag that restricts transposition. The $2.8 \AA$ crystal structure of p18m reveals an all $\alpha$-helical protein related to mammalian and insect ARC proteins. p18m retains the capacity to dimerise in solution and the crystal structures reveal two exclusive dimer interfaces. We probe our findings through biophysical analysis of interface mutants as well as Ty1 transposition and p18m restriction in vivo. Our data provide insight into Ty1 Gag structure and suggest how p22/p18 might function in restriction through a blocking-of-assembly mechanism.

\footnotetext{
${ }^{1}$ Macromolecular Structure Laboratory, The Francis Crick Institute, London, UK. ${ }^{2}$ Department of Biochemistry and Molecular Biology, University of Georgia, Athens, Georgia, USA. ${ }^{3}$ Bioinformatics and Biostatistics STP, The Francis Crick Institute, London, UK. ${ }^{4}$ Retrovirus-Host Interactions Laboratory, The Francis Crick Institute, London, UK. ${ }^{5}$ Department of Infectious Disease, Imperial College London, London, UK. ${ }^{6}$ Present address: Sir William Dunn School of Pathology, University of Oxford, Oxford, UK. ${ }^{7}$ These authors contributed equally: Matthew A. Cottee, Sean L. Beckwith. ${ }^{凶}$ email: djgarf@uga.edu; ian.taylor@crick.ac.uk
} 
$\mathrm{R}$ etrotransposons replicate through a reverse transcription step and are highly prevalent in eukaryotic genomes ${ }^{1-3}$. The budding yeast $S$. cerevisiae contains members of the Ty1-copia (Ty1, Ty2, Ty4, and Ty5), and Ty3-gypsy families of LTR retrotransposons ${ }^{4}$. Tyl is the most abundant element in many strains with about 32 full-length copies in the reference strain S288C 5,6. LTRs flank the $5^{\prime}$ and $3^{\prime}$ ends of the $5.9 \mathrm{~kb}$ genomic sequence that contains $G A G$ and $P O L$ genes. After transcription from the $5^{\prime}$ - to $3^{\prime}$ - LTR, GAG encodes Gag-p49, which is analogous to retroviral Gag and provides both capsid (CA) packaging and nucleocapsid (NC) nucleic acid chaperone functions. $P O L$ encodes the protease (PR), integrase (IN), and reverse transcriptase (RT) enzymes, all of which are required for Tyl replication and integration.

Ty1 replication and integration is similar to that of retroviruses but occurs intracellularly, and transposition is not infectious ${ }^{7}$. After transcription by RNA polymerase II, Tyl genomic RNA is exported to the cytoplasm and two proteins are translated, Gagp49 and Gag-Pol-p199, produced from +1 translational frameshift between the GAG and POL genes ${ }^{8}$. Gag-p49 and Gag-Polp199 assemble along with the incorporation of dimeric Ty1 genomic $\mathrm{RNA}^{9}$ at cytoplasmic foci called T-bodies or retrosomes to form virus-like particles (VLPs) ${ }^{5,10-12}$. Assembly of the immature VLP induces self-cleavage and release of PR, encoded in Gag-Pol-p199 and further cleavage of Gag-Pol-p199 then releases the IN and RT enzymes ${ }^{5}$. PR also cleaves Gag-p49 towards the C-terminus producing Gag-p45, the mature capsid and nucleic acid chaperone protein ${ }^{13-15}$, forming mature Ty1 VLPs, which perform similar functions to the viral core in retrovirus particles. Reverse transcription of Tyl genomic RNA occurs within the VLP and the Tyl cDNA-IN pre-integration complex is imported into the nucleus and integrated mainly at sites upstream of RNA polymerase III transcribed genes ${ }^{16,17}$.

Uncontrolled retrotransposition in the genome of any organism would be highly detrimental through the effects of integration in or near active genes causing mutation, unregulated expression, and genome instability ${ }^{18-21}$. Therefore, in higher eukaryotes mechanisms including RNAi pathways along with SAMHD1 and APOBEC restriction factors prevent excessive transposition ${ }^{22,23}$. In $S$. cerevisiae, these defence systems are not present and uncontrolled Ty1 retrotransposition is restricted by a separate mechanism, referred to as copy number control (CNC) ${ }^{24-26}$. A sub-genomic transcript initiated from within the Tyl GAG gene, Tyli, contains the C-terminal half of GAG as well as POL. A $22 \mathrm{kDa}$ protein is translated from either of two alternative start codons, AUG1 or AUG2 found proximal to the $5^{\prime}$ end of the Tyli transcript ${ }^{25,27}$. Thus, p22 is identical to the C-terminal half of Gag-p49 and is processed at the C-terminus by PR to generate a mature protein $\mathrm{p} 18$, that is identical to the C-terminal half of mature capsid Gag-p45 25,27 . Both p22 and p18 can restrict Ty1 retrotransposition, and so they constitute a self-encoded restriction factor. Although biochemical and fluorescence microscopy studies suggest that p22 associates with Gag-p45 $25,27,28$, the mechanism of $\mathrm{p} 22 / \mathrm{p} 18$ restriction of Ty1 retrotransposition is less clear. Multiple mechanisms have been proposed involving inhibition of different stages in the replication cycle ${ }^{24}$. These include inhibition of Gag-p45 nucleic acid chaperone function ${ }^{27}$, disruption of Ty1-Gag retrosome formation, and prevention of VLP assembly $^{25}$.

Crystallographic and cryo-electron microscopy structural studies of retroviral Gag and CA have revealed how monomers first assemble into hexamers ${ }^{29-31}$, and when combined with CA pentamers can further assemble into closed Fullerene shell structures ${ }^{32-37}$ that are found in retroviral cores. The relative contribution from $\mathrm{N}$ - and C-terminal CA domains (NTD/CTD) in capsid assembly also varies in different retroviruses $29,38-40$.
Structural studies of Ty3 Gag as well as the ARC protein from Drosophila (dARC) have revealed how the same hexameric and pentameric building blocks are utilised in VLP shell assembly ${ }^{41-43}$. However, to date no high-resolution structures of Ty1 Gag-p45 or p22/p18, or any member of the Ty1-Copia retrotransposon family are available to help define parameters of Ty1 VLP assembly or the structural basis of CNC.

Here we report the $2.8 \AA$ crystal structure of a minimal p18 from Ty1-Gag (p18m) that is able to restrict Tyl transposition. The structure comprises an all $\alpha$-helical domain related to that observed in the CA-CTD of the yeast Ty3 retrotransposon, ARC proteins, and orthoretroviruses. The crystal structure contains two independent p18m dimer interfaces and analytical ultracentrifugation reveals a tight dimer that can further oligomerise. We test the significance of our structural findings using mutagenesis combined with biophysical studies in vitro and transposition and CNC analyses in vivo. Our work demonstrates the importance of an evolutionarily conserved transposon and retroviral CA-CTD interface and provides insight into a unified mechanism of Tyl CNC.

\section{Results}

Defining a minimal Tyl Gag p18 restriction domain. Ty1 GAG contains several regions based on structure predictions, biochemical and genetic analyses, and phylogenomic comparisons (Fig. 1a). In particular, predicted helical regions, are contained within the CNC-resistance $\left(\mathrm{CNC}^{\mathrm{R}}\right)$ and UBN2/Retrotran_gag_2 PFAM domains, respectively ${ }^{28}$. The helical regions also correspond to the CA-NTD and CA-CTD of LTR retrotransposons and retroviruses ${ }^{41}$. Specific $\mathrm{CNC}^{\mathrm{R}} / \mathrm{CA}-\mathrm{NTD}$ amino acid substitutions confer resistance to the p22 restriction factor, and $\mathrm{UBN} 2 / \mathrm{CA}-\mathrm{CTD}$ is within $\mathrm{p} 22 . \mathrm{CNC}^{\mathrm{R}}$ mutations also occur in the UBN2/CA-CTD domain ${ }^{28}$.

Segments within the p22 and p18 coding sequence (Fig. 1b) were expressed ectopically and assessed for their capacity to restrict Ty1 retromobility. The constructs (Fig. 1b) were chosen based on the previously defined AUG1 and AUG2 initiation codons and on analysis of secondary structure predictions for Ty1-Gag 25,27,28. Constructs also contained a hexa-histidine tag to aid in protein detection and purification. p18 constructs were inducibly co-expressed with a Tyl element containing the his3-AI indicator gene $\mathrm{e}^{44}$ to determine the effect on retromobility (Fig. 1c). All p18 deletion constructs were also assessed by Western blot (Fig. 1d). As expected, initiation at AUG1 (p18m $\mathrm{m}_{\mathrm{AUG} 1}$ ) resulted in much higher levels of expression in yeast than initiation at AUG2 $\left(\mathrm{p} 18 \mathrm{~m}_{\mathrm{AUG} 2}\right)^{27,45}$. Constructs were tested for their capacity to inhibit Tyl mobility in a qualitative plating assay, and these data showed that truncated tagged p18 proteins were still able to restrict Tyl transposition (Fig. 1e). Quantitative mobility assays (Fig. 1f and Supplementary Table 1) revealed that a fragment containing AUG1 to residue 355 of 18 retained potent restriction of Ty1, comparable to full-length p18. The restriction is also apparent with $\mathrm{p} 18 \mathrm{~m}_{\mathrm{AUG} 2}$ but at a lower level, correlating with the reduced expression. The co-expression results show that regions of Gag required for CNC comprise residues M249-N355 in p18m $\mathrm{m}_{\mathrm{AUG} 1}$ or M259-N355 in p18m $\mathrm{m}_{\mathrm{AUG} 2}$ and imply that the nucleic acid chaperone domain of Gag (Fig. 1a) is not required for CNC.

Structure of the Tyl Gag p18m domain. We determined the crystal structures of $\mathrm{p} 18 \mathrm{~m}_{\mathrm{AUG} 1}$ and $\mathrm{p} 18 \mathrm{~m}_{\mathrm{AUG} 2}$ expressed in $E$. coli. The $\mathrm{p} 18 \mathrm{~m}_{\mathrm{AUG} 2}$ structure was solved by multi-wavelength anomalous diffraction (MAD) using crystals of Se-Met substituted protein. The p18m $\mathrm{m}_{\mathrm{AUG}}$ structure was solved by molecular replacement using the $18 \mathrm{~m}_{\mathrm{AUG} 2}$ structure as a search 
a

Ty1 retrotransposon

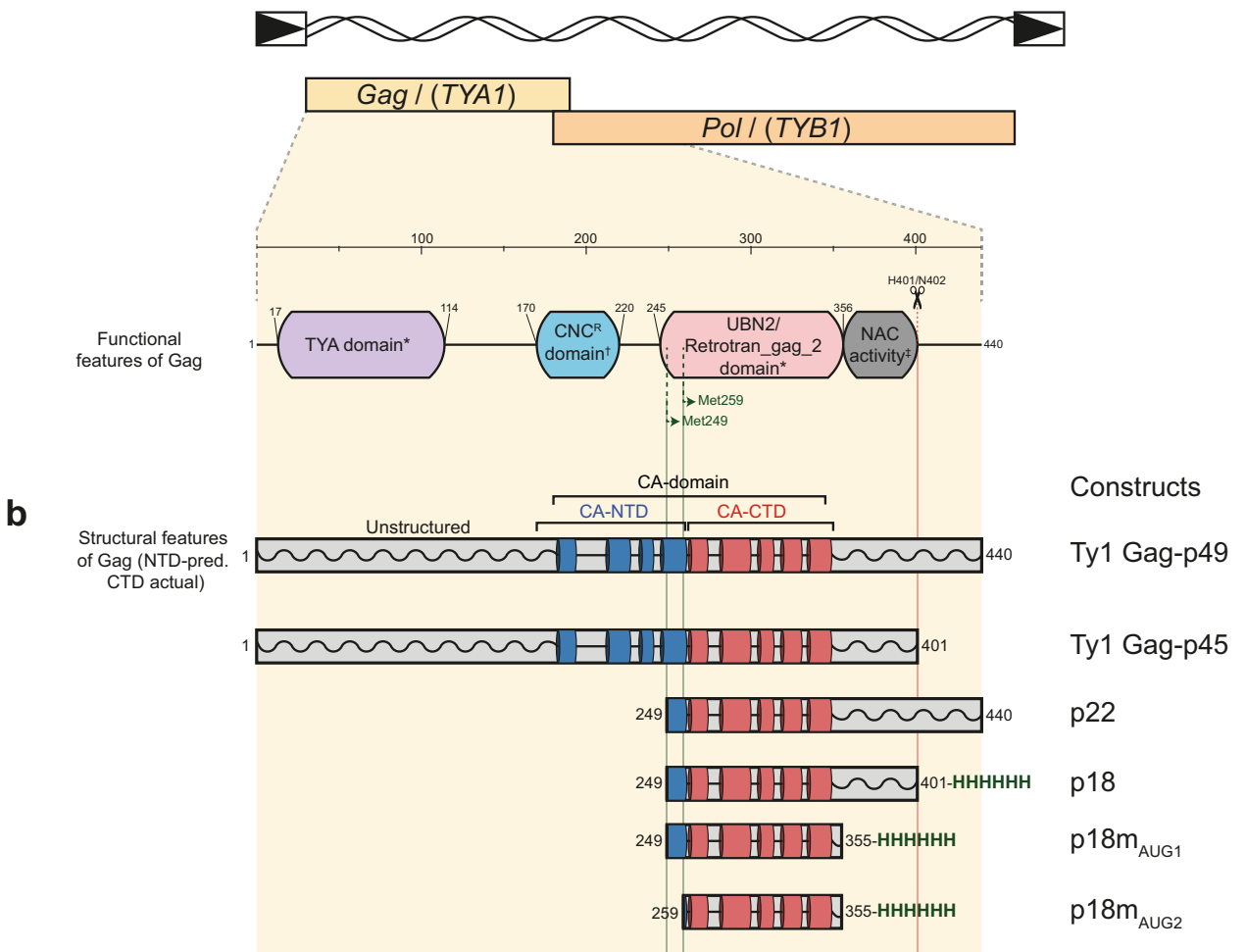

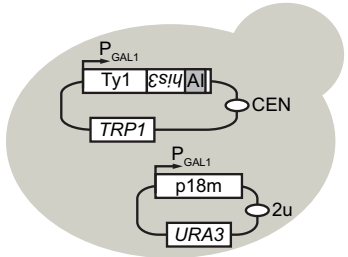

d

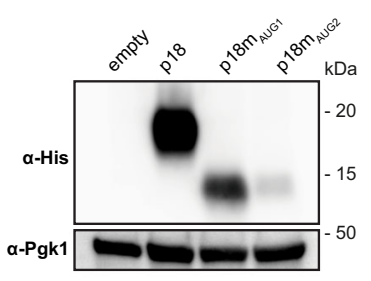

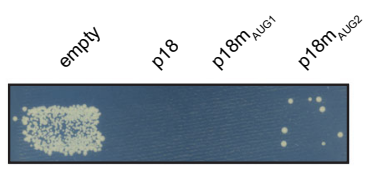

f

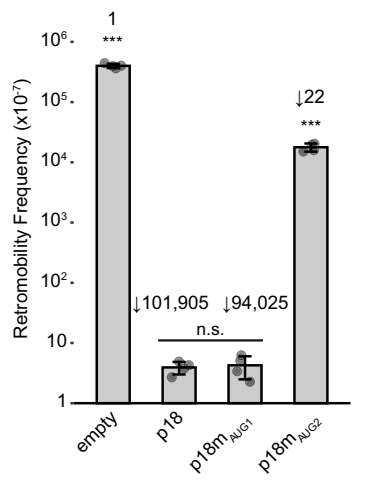

additional density for residues 249 to 259 that constitute the additional N-terminal sequence difference between AUG1 and AUG2. Therefore, we consider both structures to be identical, and define $\mathrm{p} 18 \mathrm{~m}$ as residues $\mathrm{M} 259-\mathrm{N} 355$ (p18m $\mathrm{mUG}_{2}$ ) and representing the minimal domain required for CNC activity.

Structural similarity with ARC and retroviral CA. Structural similarity searches of $\mathrm{p} 18 \mathrm{~m}$ using the DALI search engine ${ }^{46}$ identified Drosophila and mammalian ARC CA-CTD structures as top hits with DALI $\mathrm{Z}$ scores ranging from 8.6-7.0 (Supplementary Fig. 1). The CTD of Ty3 CA also provided a strong match with a DALI Z score of 7.7. These topological and structural similarities reflect the evolutionary relationship between retrotransposons and the exapted ARC proteins. There were also 
Fig. 1 Transposition assays define the minimal fragment for p22/p18 activity. a Schematic of the Ty1 retrotransposon, highlighting the TY1A GAG and TY1B POL genes. The functional regions and cleavage site of Gag identified by genetic analysis are highlighted below, ${ }^{*} P F A M$ domains, ${ }^{\dagger} C N C^{R}$ domain ${ }^{28}$, FAC originally defined as residues $299-401^{15}$ redefined by this study as residues 356-401. The Met249 and Met259 translational starts that initiate from the AUG1 and AUG2 codons are indicated. b Schematic of p22/p18 constructs and structural features of Gag. Gag-p49 and p22 undergo proteolytic maturation into Gag-p45 and p18, respectively. A restrictive fragment terminating at residue $355(\mathrm{p} 18 \mathrm{~m})$ is defined in this study and all p18 constructs also contain a C-terminal hexa-histidine tag. c Schematic illustrating the two plasmids used to co-express the Ty1 transposon and the p18 restriction factor. The expression of each is driven by a galactose-inducible promoter from the GAL1 gene. Ty1 contains the his3-Al retromobility indicator gene; histidine prototrophy requires retromobility. d Western blot of p18 fragment expression. Protein extracts of galactose-induced cells were immunoblotted with an anti-hexa-histidine antibody. Pgk1 serves as a loading control. Strains used: empty (DG3739), p18 (DG4162), p18m AUG1 (DG4147), p18m AUG2 (DG4146). Migration of molecular weight standards is shown alongside the immunoblot. A representative image of at least 3 replicates is shown, images of entire gel immunoblots are provided in the Source Data file. e Qualitative mobility assay showing CNC effect of p18 constructs on Ty1 retromobility. Cells were galactose-induced; growth on selective media lacking histidine indicates a retromobility event. A representative image of at least 3 replicates is shown. f Quantitative mobility assay of galactose-induced cells. Each bar represents the mean of the four independent measurements displayed as points. The error bar centre represents the mean of the four measurements and the error bar extent \pm the standard deviation. Fold-change compared with empty vector is indicated above the bars. Significance is calculated from a two-sided Student's $t$-test compared with p18 (n.s not significant, ${ }^{\star \star \star} p<0.001$. Exact $p$-values are provided in Supplementary Table 1, source data is provided in the Source Data file.

matches with CA-NTDs from ARC proteins (DALI Z score 5.8) and Ty3 (DALI $Z$ score 6.1), supporting the notion that tandem domains of CA arose as the result of a gene duplication event ${ }^{47}$. The closest matching retroviral structure was with the CA-CTD from the gamma-retrovirus MLV with a DALI Z score of 7.8. Weaker matches with the CA-CTD and CA-NTD from HIV-1 and the CA-CTD from the endogenous retrovirus HERV-K (DALI Z-scores 5.3-4.9) demonstrate the more distant relationship between retrotransposon and retroviral CAs.

The p18m dimer interfaces. Inspection of the $\mathrm{p} 18 \mathrm{~m}$ crystal structures revealed three monomers in the ASU, each forming two dimer interfaces (Fig. 2c). In the first (Dimer-1), the exposed surfaces of $\alpha 1$ and $\alpha 3$ pack against $\alpha 1^{\prime}$ and $\alpha 3$ ' of the opposing monomer (Fig. 2d). The entire dimer interface encompasses $773 \AA^{2}$ of buried surface and is defined by largely hydrophobic interactions with contributions from sidechain packing of A266, I269, V270, A273 on $\alpha 1$ and I302, I304, V308, and L312 on a3 and the preceding interspersing $a 2-\alpha 3$ loop that form a continuous apolar network with I269 and A273 at its centre (Fig. 2d). In addition, E265 and K307 at the N-termini of a 1 and $\alpha 3$, respectively, make a salt bridge interaction at the periphery of the interface that further stabilises the dimer. The extent and hydrophobic nature of interactions within this homodimer interface suggests the dimer constitutes a relatively stable or obligate structure. Moreover, this interface is conserved amongst dARC and retroviral CA-CTD structures (Supplementary Figure 2) that also comprise an equivalent hydrophobic core.

To analyse surface conservation at this interface, we conducted a multiple sequence alignment of $125 \mathrm{Ty} 1 \mathrm{Gag}$ sequences found in Saccharomyces genomes. The alignment (Supplementary Fig. 3a) was mapped onto the structure using the Consurf server ${ }^{48,49}$ and revealed that residues making significant contributions to the interface (especially the highly hydrophobic patch formed by I269/I302/I304/V308 and salt-bridging residues K307/E265) were near-universally conserved (Supplementary Fig. 3b, c). Other interface residues were substituted for similar residues. Our analyses suggest that this hydrophobic dimerisation interface is a conserved feature of Tyl Gag found throughout Saccharomyces and is similar in nature to CTD dimers from divergent CA proteins.

In the second interface (Dimer-2), residues on the outer surface of $\alpha 4$ and $\alpha 5$ pack against $\alpha 4^{\prime}$ and $a 5^{\prime}$ of the opposing monomer (Fig. 2e). This dimer interface encompassed $690 \AA^{2}$ of buried surface and comprises a hydrophobic network with sidechain packing of F323, Y326, and T327 on a 4 with A345 and I346 on a5. Notably, the Dimer-2 interface is not sequence conserved in
Ty3, dARC, or retroviral CA. However, upon structural superposition of $\mathrm{p} 18 \mathrm{~m}$ with Ty3 CA in assembled shells ${ }^{41}$ (PDB ID 6R24; DALI Z score 7.7), it is apparent that not only do the $a 4$ and $a 5$ helices of $18 \mathrm{~m}$ and those of the Ty3 CA-CTD align well at the level of tertiary structure, they are located at the local interpentamer and inter-hexamer 3-fold axes in Ty3 (Supplementary Fig. 4) and by inference at the equivalent 3-fold axes of Ty1 particles ${ }^{50}$. These similarities raise the possibility that Dimer-2 in the p18m crystal structures is a remnant of the trimer formed in Tyl CA assembled shells but that the crystal packing in this case does not allow for the formation of this trimer interaction.

p18m self-associates in solution. Given two different dimer interfaces were observed in the p18m crystal structure, the solution molecular mass, conformation, and self-association properties of p18m were examined using solution hydrodynamic methods. Initial Size Exclusion Chromatography coupled MultiAngle Laser Light Scattering (SEC-MALLS) analysis was performed with protein concentrations ranging from $100-400 \mu \mathrm{M}$. These data yielded a solution molecular weight of $26 \mathrm{kDa}$ for p18m but with an indication of the further weak association at the highest concentrations employed (Fig. 3a). The p18m sequencederived molecular weight is $12.2 \mathrm{kDa}$. Therefore, $\mathrm{p} 18 \mathrm{~m}$ forms strong dimers in solution with some tendency for further selfassociation at higher concentrations.

To further understand $\mathrm{p} 18 \mathrm{~m}$ oligomerisation, sedimentation velocity (SV-AUC), and sedimentation equilibrium (SE-AUC) analytical ultracentrifugation were employed to analyse $\mathrm{p} 18 \mathrm{~m}$ hydrodynamic properties (Supplementary Table 3). Sedimentation velocity data for $\mathrm{p} 18 \mathrm{~m}$ over a concentration range of $42-165 \mu \mathrm{M}$ and analysed using the $\mathrm{C}(\mathrm{S})$ continuous sedimentation coefficient distribution function revealed two predominant species (Fig. 3b). All p18m samples contained a slow component with $S_{20, w}$ of $2.32 \pm 0.03$. However, at increasing protein concentration a fast component was detected with a sedimentation coefficient that increased from $S_{20, w}$ of 2.90 at $82 \mu \mathrm{M}$ to $S_{20, w}$ of 3.09 at $165 \mu \mathrm{M}$. Concentration dependency was also apparent by evaluation of the weight average sedimentation coefficient, obtained by integration of the entire envelope of the $C(S)$ function, which also showed an increase with increasing concentration (Supplementary Table 3).

The solution molecular weight of the slow and fast components was determined by combining the sedimentation coefficients with the best fit frictional ratio $\left(f / f_{0}\right)$ from the $\mathrm{C}(\mathrm{S})$ analysis. This gave $21.9 \pm 0.7 \mathrm{kDa}$ for the slow species, close to the formula mass of a p18m dimer, and a concentration-dependent value of $30.0-33.7 \mathrm{kDa}$ for the fast species. These data show that $\mathrm{p} 18 \mathrm{~m}$ 
a

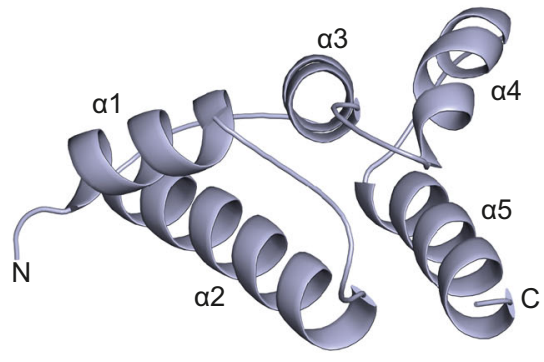

b

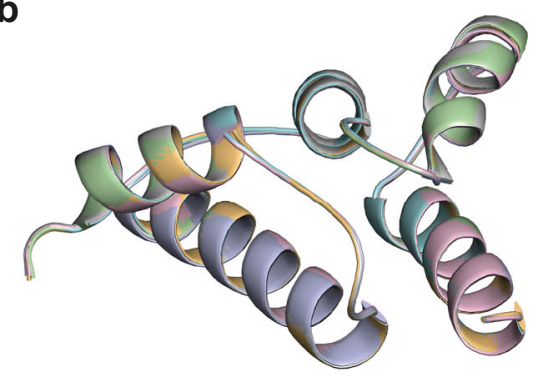

C

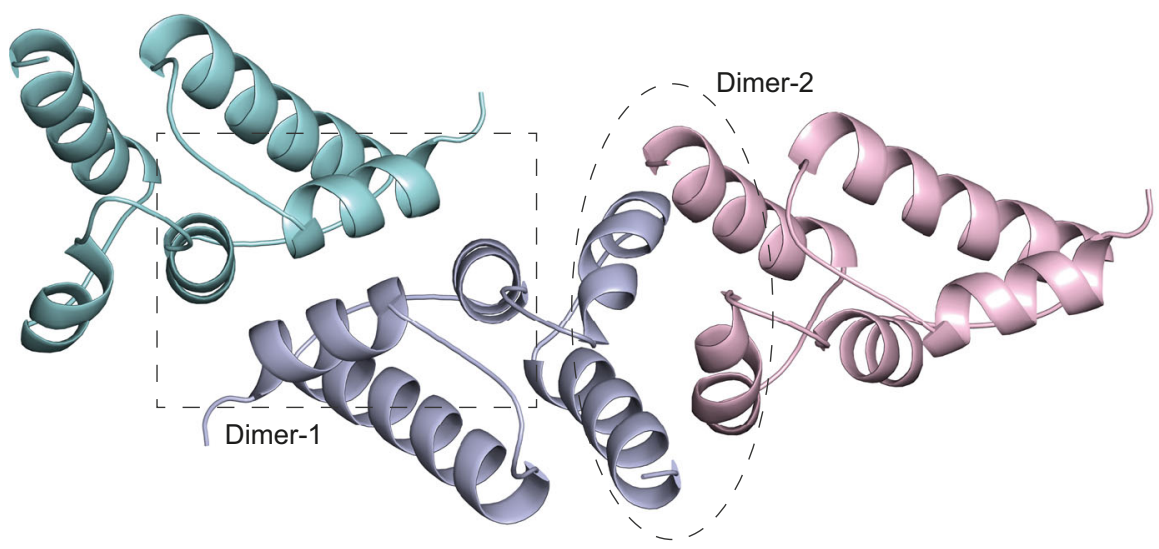

d

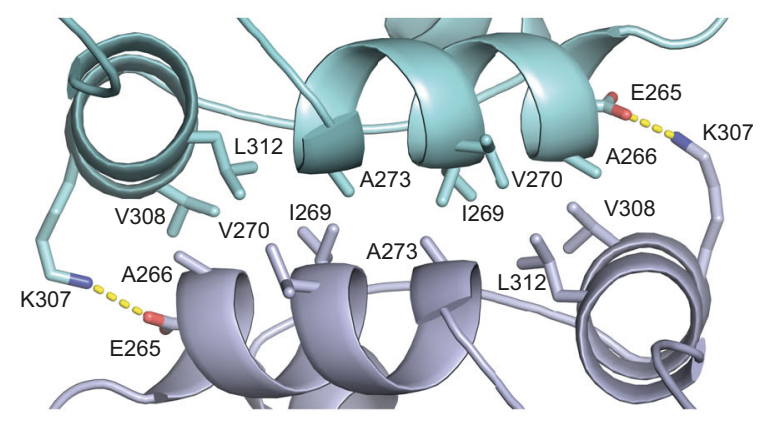

e

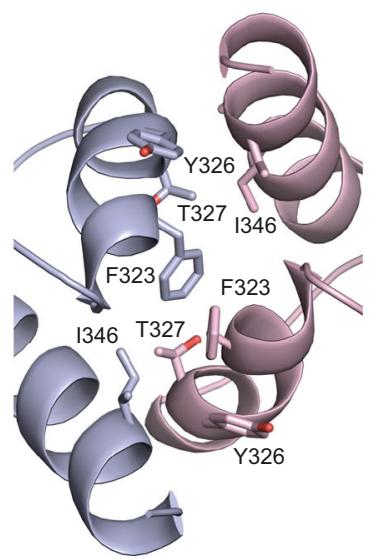

Fig. 2 Crystal structure of $\mathbf{p} \mathbf{1 8} \mathbf{m}$. a Crystal structure of p18m monomer, the protein backbone is shown in cartoon representation. The five $\alpha$-helices in the structure are labelled sequentially from the $\mathrm{N}$ - to C-terminus. b 3D structural alignment of all six copies of p18m present in the AUG1 and AUG2 structures. The view is the same as in $\mathbf{a}$. Individual monomers are shown in cartoon representation coloured light-blue, cyan, pink, orange, grey, and green. Structures were aligned using backbone $\mathrm{C} \alpha$ atoms (RMSD $0.16 \pm 0.04 \AA$ over $82 \mathrm{C} \alpha$ ). c The trimer asymmetric unit of the p18m crystal structure. The three p18m protomers are shown in cartoon representation coloured cyan, light-blue and pink respectively. The dimer interfaces between protomers (Dimer-1 and Dimer-2) are highlighted with the grey box and ellipse respectively. $\mathbf{d}$ Close-up view of the Dimer-1 interface, the box-highlighted region in (c). Residues on helices $\alpha 1$ and $\alpha 3$ that make apolar and salt bridge interactions are shown in stick representation. The E265 and K307 salt bridge is indicated by the dashed lines. e Close-up view of the Dimer- 2 interface, the ellipse-highlighted region in (c). Residues on helices $\alpha 4$ and $\alpha 5$ that make the hydrophobic interactions are shown in stick representation.

comprises a stable $2.32 \mathrm{~S}$ species with a molecular weight consistent with a p18m dimer, but that this dimeric species can also further self-associate into larger oligomers, consistent with the behaviour observed by MALLS. To further characterise p18m self-association, multispeed SE-AUC studies at varying protein concentrations were carried out. Typical equilibrium distributions recorded at the three speeds are presented in Fig. 3c. Analysis of individual gradient profiles using a simple individual species model showed there was a strong concentration dependency of the molecular weight ranging from 31.6 to $39.6 \mathrm{kDa}$ and poor fitting of the data. Given our observations in the sedimentation velocity experiments, the data were fitted globally using a monomer-dimer-tetramer model (Fig. $3 \mathrm{c}$ and Supplementary Table 3). The application of this model gave a much-improved best fit that comprised $\mathrm{p} 18 \mathrm{~m}$ monomers in a tightly associating monomer-dimer equilibrium, $K_{D}^{(1-2)}$ of $0.73 \mu \mathrm{M}$, together with a weakly associating dimer-tetramer equilibrium, $K_{D}(2-4)$ of $43.2 \mu \mathrm{M}$.

Dimer-2 is required for transposition but not restriction. To test the functional significance of $\mathrm{p} 18 \mathrm{~m}$ self-association, we first introduced an F323S mutation at the Dimer-2 interface to disrupt 
a

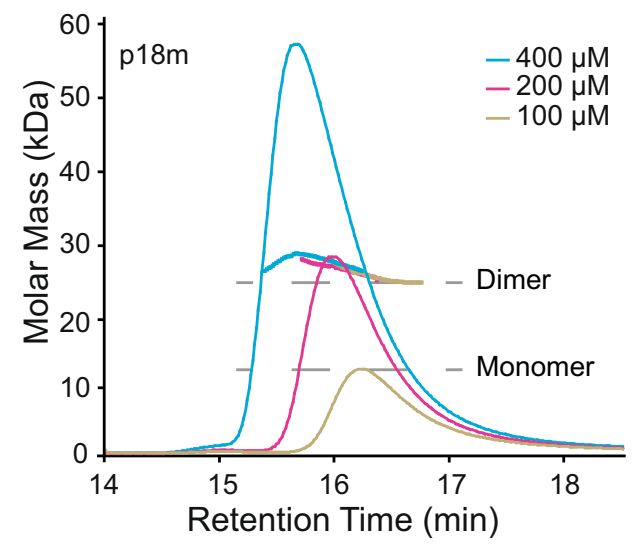

C
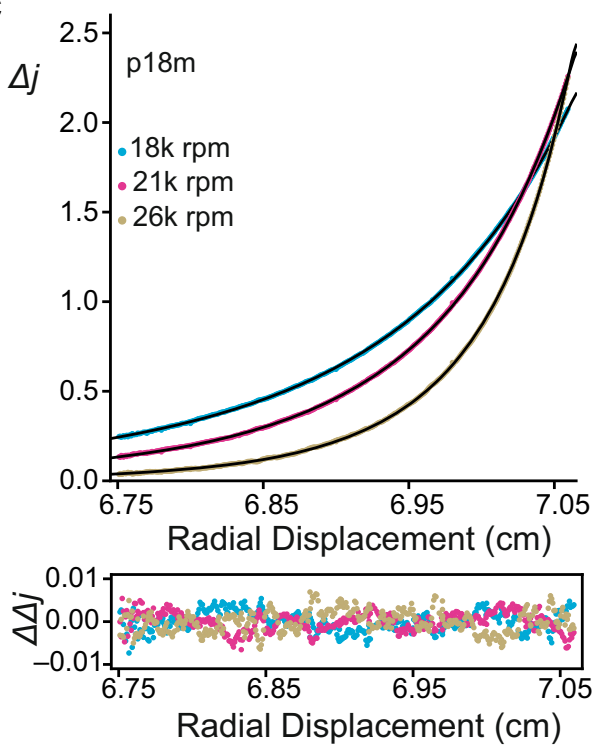

e

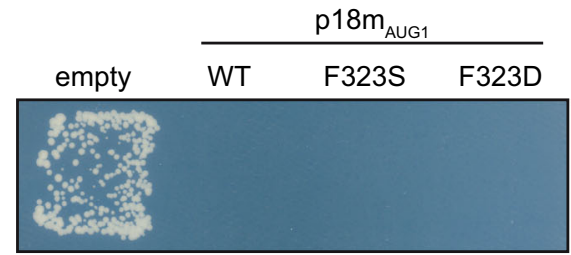

f

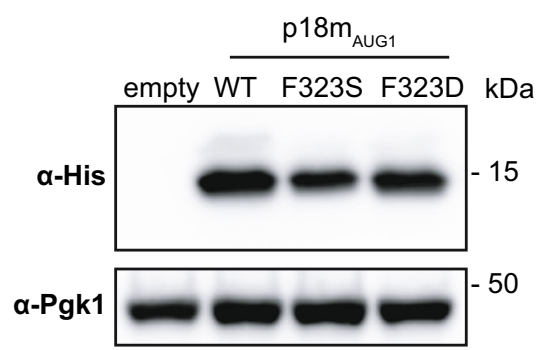

a4-a4 hydrophobic interactions. The introduction of this polar sidechain had minimal effects on protein expression. Assessment of the p18m-F323S solution oligomeric state by SEC-MALLS yielded a solution molecular weight of $26 \mathrm{kDa}$ and showed that further higher-order association was suppressed over the concentration range tested (100-400 $\mu \mathrm{M})$ (Fig. 3d). This suggests that b

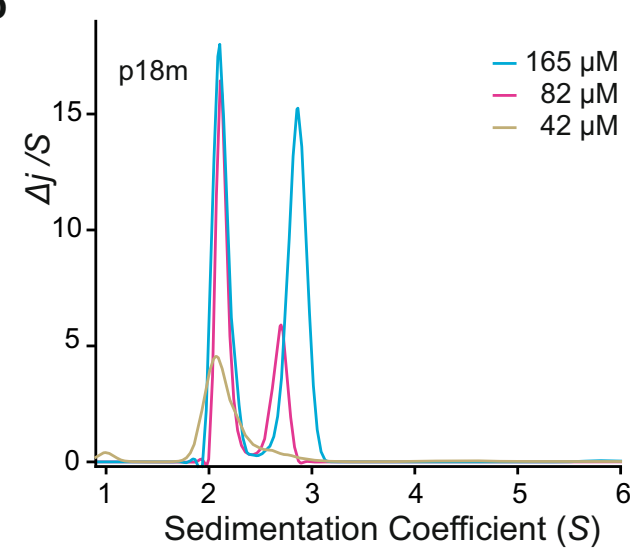

d

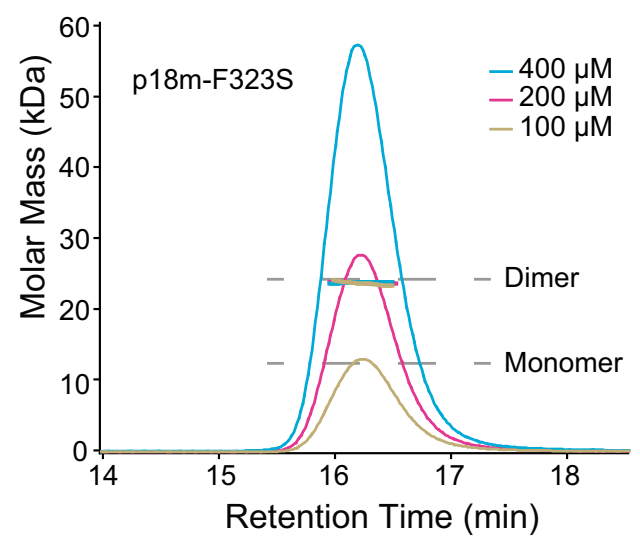

g

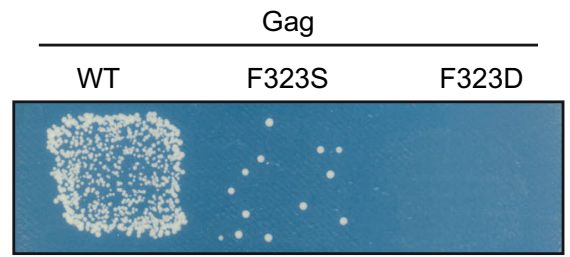

h

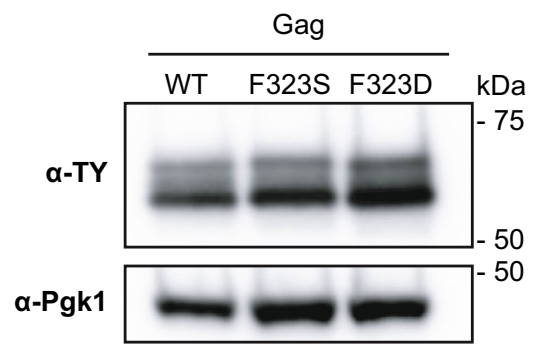

the Dimer-2 interface mediates only the weak higher-order association whilst the Dimer-1 interface is responsible for forming the strong dimer we observe in the solution.

In yeast, disruption of the Dimer-2 interface by the F323S or more severe charge-clash F323D mutation in p18m 18 AUG did not markedly impair restriction or reduce protein expression (Fig. 3e, 
Fig. 3 p18m self-associates in solution. a, d SEC-MALLS analysis of p18m and Dimer-2 interface-mutant p18m-F323S. The sample loading concentrations were $400 \mu \mathrm{M}$ (cyan), $200 \mu \mathrm{M}$ (magenta), and $100 \mu \mathrm{M}$ (wheat). The differential refractive index (dRI) is plotted against column retention time and the molar mass, determined at $1 \mathrm{~s}$ intervals throughout the elution of each peak, is plotted as points. The p18m monomer and dimer molecular mass is indicated with the grey dashed lines. b C (S) distributions were derived from sedimentation velocity data recorded from p18m at $42 \mu \mathrm{M}$ (wheat), $82 \mu \mathrm{M}$ (magenta), and $165 \mu \mathrm{M}$ (cyan). The curves are the distribution of the sedimentation coefficients that best fit the sedimentation data (RMSD 0.004-0.016), see also Supplementary Table 3. c Multispeed sedimentation equilibrium profiles determined from interference data collected on p18m at $30 \mu \mathrm{M}$. Data were recorded at the speeds indicated. The solid lines represent the global best fit to the data using a monomer-dimer-tetramer model $\left(K_{D}{ }^{(1-2)}=0.73 \mu \mathrm{M}, K_{D}{ }^{(2-4)}=43.2 \mu \mathrm{M}\right.$; reduced $\left.\chi^{2}=2.22\right)$. The lower panel shows the residuals to the fit, see also Supplementary Table 3. Source data for $\mathbf{b}$ and $\mathbf{c}$ are provided in the Source Data file. e, $\mathbf{g}$ Effect of $18 \mathrm{~m}_{\mathrm{AUG}}$ and Gag mutations on Ty1 mobility. Growth on selective media; His ${ }^{+}$cells indicate a retromobility event. A representative image of at least 3 replicates is shown. p18m $m_{A U G 1}$ strains used: empty (DG3739), WT (DG4147), F323S (DG4350), F323D (DG4351). Gag strains used: WT (DG3735), F323S (DG4348), F323D (DG4349). f, h Protein extracts prepared from galactoseinduced yeast cells expressing the indicated $18 \mathrm{~m}_{\mathrm{AUG} 1}$ or Gag mutants were immunoblotted with hexa-histidine antibody to detect p18m $\mathrm{m}_{\mathrm{AUG}}$ or TY-tag antibody to detect Gag. Pgk1 serves as a loading control. Migration of molecular weight standards is shown alongside the immunoblots. A representative image of at least 3 replicates is shown. Images of the whole gel immunoblots are provided in the Source Data file.

f), supporting the notion that the Dimer-2 interface can be modified and $\mathrm{p} 18 \mathrm{~m}$ still retain function in vivo. By contrast, whilst the introduction of F323S or F323D into Ty1 GAG did not affect Gag expression compared with WT, both substitutions dramatically reduced retromobility with F323D having the severest effect (Fig. 3g, h).

Dimer-1 Gag assembly mutations affect Ty1 mobility and CNC. We investigated the functional significance of the Dimer-1 hydrophobic interface by targeted mutagenesis. We first made interface-disruptive mutations that were polar I269S and A273Q, charged I269K and A273D, or increased hydrophobic-bulk, A273M. All resulted in the loss of protein stability/solubility, as judged by our inability to recover and purify soluble proteins when expressed in E. coli. Additionally, the introduction of I269S or A273M mutations in either p18m or Gag dramatically reduced protein accumulation in yeast (Supplementary Table 4).

We also made random mutations at the key Dimer-1 interface residues I269 and A273 and characterised them in the context of GAG within a complete Tyl element. An NNK mutagenesis strategy $^{51}$ (see Methods) was applied to randomize all 20 amino acid codons at I269 and A273 while reducing premature stop codons. Results from both NNK mutagenesis (Supplementary Fig. 5) and targeted mutagenesis (Supplementary Table 4) fit the trend that like-for-like mutations are tolerated but nonconservative mutations interfere with Gag accumulation and transposition. Of the tolerated conservative substitutions, we characterized the effects of $\mathrm{A} 273 \mathrm{~V}$, previously identified as an escape mutant from p22-based $\mathrm{CNC}^{28}$, and I269F. In qualitative plate assays, galactose-induced expression of Tyl Gag-A273V and Gag-I269F showed levels of transposition that were indistinguishable from WT Gag (Fig. 4a) and expressed at similar levels (Fig. 4b). Quantitative mobility assays revealed only small changes in Ty1 movement (Fig. 4c and Supplementary Table 1), indicating Gag-A273V and Gag-I269F retain the capacity to support transposition.

We characterised Dimer-1 interface mutations A273V and $\mathrm{I} 269 \mathrm{~F}$ in the context of $\mathrm{p} 18 \mathrm{~m}_{\mathrm{AUG} 1}$ to examine their ability to restrict WT Ty1. The p18m-A273V and p18m-I269F mutants were well-expressed as monitored by inducible co-expression of p18m and Ty1 and strongly inhibited Ty1 mobility in qualitative assays (Fig. $4 \mathrm{~d}$, e). They restricted Tyl transposition by $10^{5}$-fold, indistinguishable from that of p18m (Fig. $4 \mathrm{f}$ and Supplementary Table 1). These data further support the idea that the $\mathrm{p} 18 \mathrm{~m}$ Dimer-1 interface is a requirement for protein folding/structural integrity and likely forms a key building block of Ty1 particle assembly. As a result, only conservative mutations that do not perturb the Dimer-1 hydrophobic network are tolerated and able to retain both $\mathrm{p} 18 \mathrm{~m}$ and Gag functionality.
Self-association and stability of $\mathbf{p} \mathbf{1 8 m}$ interface mutants. Since the $\mathrm{I} 269 \mathrm{~F}$ and $\mathrm{A} 273 \mathrm{~V}$ mutants were tolerated in vivo, $\mathrm{p} 18 \mathrm{~m}_{\mathrm{AUG}}$ mutants were examined for their effects on protein oligomerisation and stability. A SEC-MALLS analysis performed at increasing protein concentration $(100-400 \mu \mathrm{M})$ yielded a molecular weight of $26 \mathrm{kDa}$ for both p18m-A273V and p18m-I269F, consistent with the dimer molecular weight. Further weak higherorder self-association at the highest concentrations employed was also evident (Fig. 5a, b). These data demonstrate that the I269F or A273V mutations are accommodated within the interface without disrupting $\mathrm{p} 18 \mathrm{~m}$ dimerisation.

To characterise the assembly properties of the $\mathrm{I} 269 \mathrm{~F}$ and A273V mutants and quantify the affinity of self-association interactions, SV- and SE-AUC measurements were undertaken (Supplementary Table 3). The best fit $\mathrm{C}(\mathrm{S})$ functions were determined from SV-AUC data recorded from $\mathrm{p} 18 \mathrm{~m}-\mathrm{A} 273 \mathrm{~V}$ and p18m-I269F over a concentration range of 30-180 $\mu \mathrm{M}$ (Fig. 5c and Supplementary Fig. 6a). For both interface mutants, as was observed with $\mathrm{p} 18 \mathrm{~m}$, the $\mathrm{C}(\mathrm{S})$ distribution contains two species, a slow component with invariant $S_{20, w}$ of $2.31 \pm 0.04(\mathrm{~A} 273 \mathrm{~V})$ and $2.30 \pm 0.05$ (I269F) and fast-moving species with a concentrationdependent $S_{20, w}(2.87-3.18)$ that constitutes a fraction of about half of the total mass at the highest concentrations measured. Analysis of the molecular weights derived from these data identifies the $2.3 \mathrm{~S}$ species as the p18m Dimer-1 and, similar to WT $18 \mathrm{~m}$, both mutants retain the capacity to further associate into the higher-order species represented by the fast component.

The affinity of self-association interactions for the interface mutants was measured using multispeed SE-AUC. Sedimentation equilibrium distributions for p18m-A273V and p18m-I269F were recorded at three speeds and varying protein concentrations (Supplementary Table 3 and Supplementary Fig. 6b, c) and the data fitted globally to a monomer-dimer-tetramer model. For both mutants, the best fit was with a tightly associating monomer-dimer equilibrium $\left(K_{D}^{(1-2)}=0.34 \mu \mathrm{M}, \mathrm{A} 273 \mathrm{~V}\right)$ and $\left(K_{D}{ }^{(1-2)}=0.74 \mu \mathrm{M}, \mathrm{I} 269 \mathrm{~F}\right)$ together with a weakly associating dimer-tetramer equilibrium $\left(K_{D}{ }^{(2-4)}=51.8 \mu \mathrm{M}, \mathrm{A} 273 \mathrm{~V}\right)$ and $\left(K_{D}{ }^{(2-4)}=45.3 \mu \mathrm{M}, \mathrm{I} 269 \mathrm{~F}\right)$. These values are largely comparable with that observed for WT p18m, confirming that A273V and I269F maintain the capacity for self-association.

p18m dimer stability. CD spectroscopy was used to analyse the secondary structure content and examine protein stability of $\mathrm{p} 18 \mathrm{~m}$ and the A273V and I269F mutants. Far UV CD spectra, $190-260 \mathrm{~nm}$, of p18m, p18m-A273V, and p18m-I269F were recorded at $10^{\circ} \mathrm{C}$ (Supplementary Fig. 6d). The spectra essentially overlay and contain a large negative differential molar extinction $(\Delta \varepsilon)$ at $222 \mathrm{~nm}$, representative of a predominantly $\alpha$-helical protein and consistent with the crystal structure. In addition, these spectra 
a

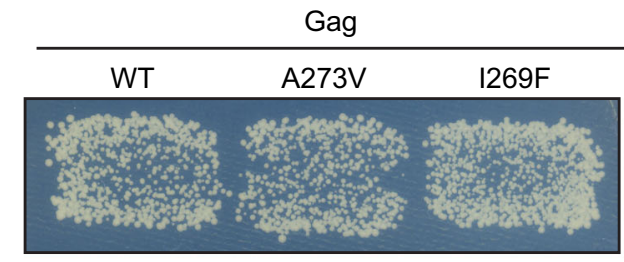

b

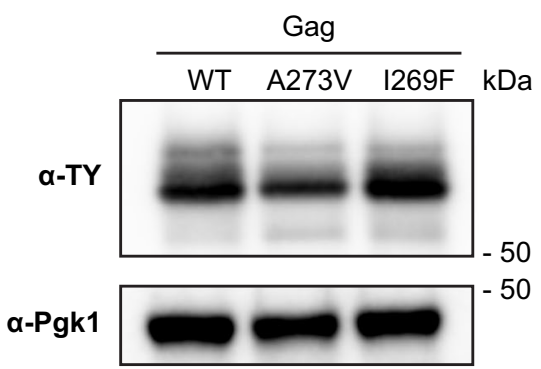

C

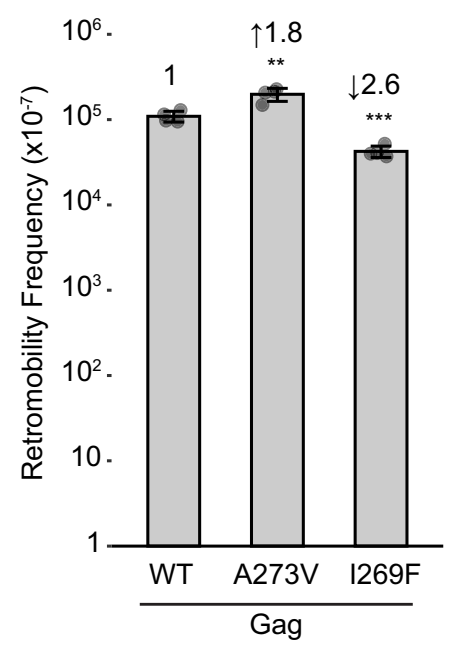

d

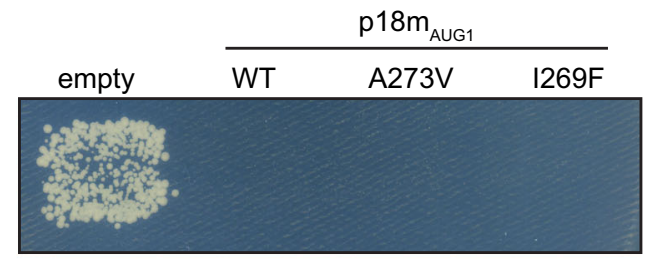

e

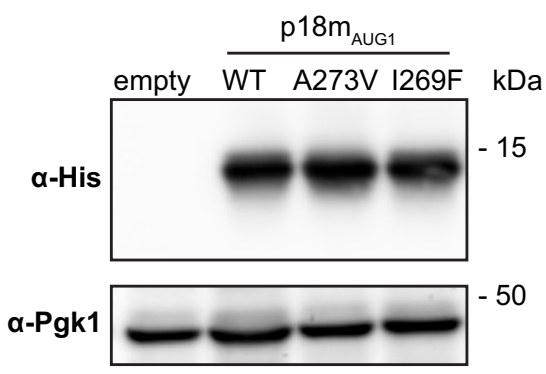

f

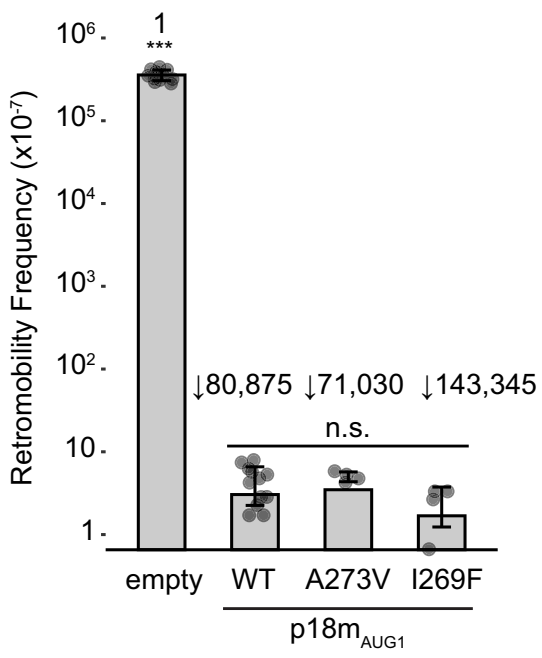

Fig. 4 Ty1 retromobility and restriction tolerates conservative interface mutants. a, $\mathbf{d}$ Effect of Gag and p18m $\mathrm{m}_{\mathrm{AUG} 1}$ mutations on Ty1 mobility. Growth on media lacking histidine indicates a retromobility event. A representative image of at least 3 replicates is shown. Gag strains used: WT (DG3735), A273V

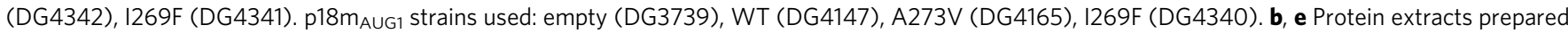
from galactose-induced yeast cells expressing the indicated Gag or p18 $\mathrm{m}_{\mathrm{A \cup G} 1}$ mutants were immunoblotted with TY-tag antibody to detect Gag or hexahistidine antibody to detect $18 \mathrm{~m}_{\mathrm{AUG}}$. Pgk1 serves as a loading control. Migration of molecular weight standards is shown alongside the immunoblots. A representative image of at least 3 replicates is shown, Images of the whole gel immunoblots are provided in the Source Data file. c, $\mathbf{f}$ Quantitative mobility assay of galactose-induced cells. Each bar represents the mean of at least four independent measurements displayed as points. The error bar centre represents the mean of the measurements and the error bar extent \pm the standard deviation. Fold-change compared with WT is indicated above the bars. Significance is calculated from a two-sided Student's $t$-test compared with WT (n.s not significant, ${ }^{\star \star} p<0.01,{ }^{\star \star \star} p<0.001$. Exact $p$-values are provided in Supplementary Table 1, See also Supplementary Table 4. Source data is provided in the Source Data file.

demonstrate that introduction of the mutations does not result in large rearrangements or loss of protein secondary structure. The stability of p18m, A273V, and I269F mutant dimers was examined by thermal denaturation monitored by far UV CD (Fig. 5d). For WT and mutants, the melting profiles were biphasic with transition midpoints $\left(T_{m}\right)$. Irreversibility of the thermal denaturation precluded a Van't Hoff analysis to detect temperature-dependent changes in $K_{D}$. However, analysis of $T_{m}$ derived from derivative plots gives values of $50.7 \pm 0.4^{\circ} \mathrm{C}$ for p18m and $45.8 \pm 0.2^{\circ} \mathrm{C}$ and $45.1 \pm 0.3{ }^{\circ} \mathrm{C}$ for $\mathrm{A} 273 \mathrm{~V}$ and $\mathrm{I} 269 \mathrm{~F}$ mutants respectively, showing that these amino acid substitutions modestly reduce protein stability. Nevertheless, the data support the notion that A273V and I269F mutations at the $18 \mathrm{~m}$ dimer interface are largely tolerated, in accord with the sedimentation data that showed only small differences in both $K_{D}{ }^{(1-2)}$ and $K_{D}{ }^{(2-4)}$. Moreover, they support the in vivo data demonstrating that when $\mathrm{A} 273 \mathrm{~V}$ or I269F mutations are introduced into Tyl-Gag, transposition is largely unaffected and when introduced into $\mathrm{p} 18 \mathrm{~m}_{\mathrm{AUG}}$, they still confer $\mathrm{CNC}$ on Ty1.

Crystal structure of p18m-A273V. To further examine the effects of the A273V mutation, we determined the crystal structure of p18m $\mathrm{m}_{\mathrm{AUG}}-\mathrm{A} 273 \mathrm{~V}$ (Supplementary Table 2). The protein crystallised in the same spacegroup as $\mathrm{p} 18 \mathrm{~m}$ with the same three copies arranged as two dimers in the ASU, but now with additional electron density for the A to V substitution on al (Fig. 5e). Superposition of the three monomers shows the backbone conformation is near identical (RMSD $=0.16 \pm 0.04 \AA$ over $78 \pm 3.6$ $\mathrm{Ca}$ ) (Fig. 5f) as was also observed with WT p18m (Fig. 2b). In 

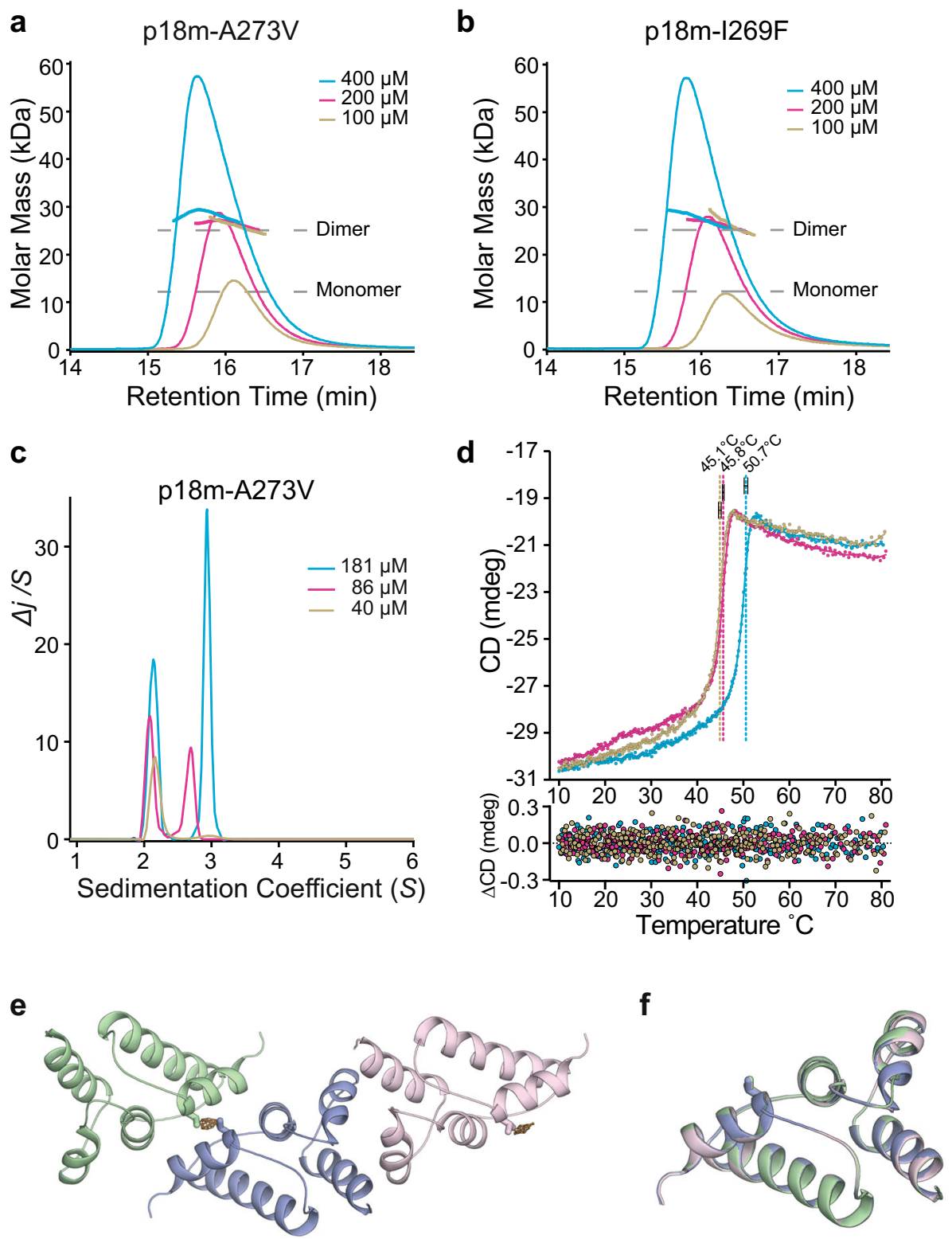

g

h
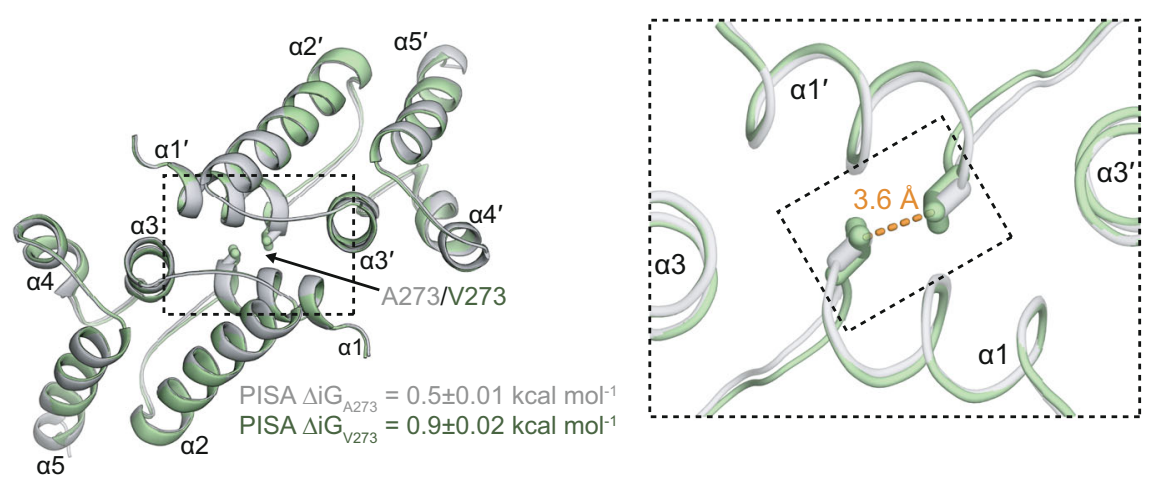

addition, the Dimer-1 interface contains the same set of apolar and $\mathrm{H}$-bond interactions as in the WT structure. The only difference is the alanine to valine substitution located at the interface centre (Fig. $5 \mathrm{~g}, \mathrm{~h}$ ). In p18m, the $\beta$-methyl groups of A273 contribute to the continuous apolar network that stabilises the dimer and packs across the interface at a favourable Van der Waals spacing of $3.6 \AA$. In p $18 \mathrm{~m}$-A273V, it is apparent that the $\gamma$-methyl groups of V273 now also form part of the continuous apolar network. However, in order to accommodate the additional methyl groups, there is a small displacement in the backbone position at the $\mathrm{C}$-terminus of $\alpha 1$ in both monomers. As a result of this shift, the V273 $\gamma$-methyl groups also pack across the dimer interface and maintain the same favourable $3.6 \AA$ Van der Waals spacing as the $\beta$-methyl groups of A273 in the p18m structure. 
Fig. 5 p18m Dimer-1 interface mutants assembly and structure. a, b SEC-MALLS analysis of p18m-A273V and p18m-I269F, sample loading concentrations; $400 \mu \mathrm{M}$ (cyan), $200 \mu \mathrm{M}$ (magenta), and $100 \mu \mathrm{M}$ (wheat). dRI is plotted against retention time. The molar mass, determined at $1 \mathrm{~s}$ intervals throughout peak elution, is plotted as points. Monomer and dimer molar masses are indicated with the dashed lines. c $C(S)$ distributions were derived from sedimentation velocity data recorded from p18m-A273V at $40 \mu \mathrm{M}$ (wheat), $86 \mu \mathrm{M}$ (magenta), and $181 \mu \mathrm{M}$ (cyan). Curves are the distribution of the sedimentation coefficients that best fit the sedimentation data (RMSD 0.009-0.025), see also Supplementary Table 3. (d) Thermal denaturation of p18m, p18m-A273V, and p18m- $1269 \mathrm{~F}$ monitored by $C D$ at $222 \mathrm{~nm}$ upon heating from $10-80^{\circ} \mathrm{C}$. Curves are the best fit spline function to the data points, dashed lines indicate the $T_{m}$ of transitions determined from the 1st derivative of the fitted curves. Error bars are standard deviations from three independent measurements. Data shown are from one representative experiment, the lower panel shows the residuals to each fit. Source data for $\mathbf{c}$ and $\mathbf{d}$ is provided in the Source Data file (e) Asymmetric unit of the p18m-A273V crystal structure. The backbone of the three p18m protomers are shown in the cartoon, coloured lime, slate and pink. The Fo-Fc map contoured at $3 \sigma$ (orange mesh), produced after molecular replacement, contains residual positive density for the valine $\gamma$-methyl groups at residue 273. $\mathbf{f} 3 \mathrm{D}$ superimposition of the 3 chains shown in (e). Structures were aligned using backbone $\mathrm{C} \alpha$ atoms ( $r m s d=0.16 \pm 0.04 \AA$ over $78 \pm 3.6 \mathrm{C} \alpha$ ). $\mathbf{g}$ Comparison of p18m (grey) and p18m-A273V (green) dimer interfaces. Structures are shown in cartoons with A273 or V273 sidechains as sticks. The PISA-calculated average solvation energy contribution to each dimer from either A273 (p18m) or V273 ( $18 \mathrm{~m}$ $\mathrm{A} 273 \mathrm{~V}$ ) are shown below. $\mathbf{h}$ Close-up view of the dimer interface, boxed in $\mathbf{g}$. Local shifts in the backbone conformation at the C-terminus of $\alpha 1$ prevent steric clashes and allow the packing of the additional $\gamma$-methyl groups of V273 preserving a favourable 3.6 $\AA$ Van der Waals distance.

Analysis of the energetic contribution from A273 or V273 to the dimer interface using PDBePISA ${ }^{52}$ also suggests that both the A273-A273 and V273-V273 interactions are favourable and that V273-V273 packing actually contributes more than the A273-A273 packing to the free energy of the overall interaction (Fig. 5g).

VLP association of $\mathbf{p 1 8 m}$ interface mutants. As full-length p18/ p22 co-sediments with Ty1 VLPs ${ }^{25,28}$ when inducibly coexpressed, we analysed the sedimentation of $\mathrm{p} 18 \mathrm{~m}_{\mathrm{AUG} 1}$ from protein extracts of yeast expressing $\mathrm{p} 18 \mathrm{~m}_{\mathrm{AUG} 1}$ and Ty1 in $7-47 \%$ continuous sucrose gradients (Fig. 6). In the absence of Ty1 expression, $\mathrm{p} 18 \mathrm{~m}_{\mathrm{AUG} 1}$ accumulated in less dense fractions at the top of the gradient (Fig. 6a). In the absence of the restriction factor, Ty1 VLPs accumulated in more dense sucrose fractions towards the bottom of the gradient, with peak fractions indicated by a bar (Fig. 6b). When Ty1 was co-expressed with full-length p18 or $\mathrm{p} 18 \mathrm{~m}_{\mathrm{AUG} 1}$, a minor fraction of the restriction factor appeared in higher density fractions (Fig, 6c, d), although the highest concentrations of 18 and $18 \mathrm{~m}_{\mathrm{AUG} 1}$ remained at the top of the gradient. This is similar to results obtained with $\mathrm{p} 22^{25,28}$. p18m $\mathrm{m}_{\mathrm{AUG1}}-\mathrm{A} 273 \mathrm{~V}$ and $\mathrm{I} 269 \mathrm{~F}$ also fractionated with VLPs (Fig. 6e, f), consistent with our data confirming their restriction activity. As previously reported ${ }^{25,28}$, the most striking effect we observe is a redistribution in the Gag fractionation pattern in the presence of restriction factor, with a shift of Tyl Gag towards the top of the gradient and an overall broadening of peak fractions (Fig. 6c-f). This redistribution is consistent with $\mathrm{p} 18 \mathrm{~m}_{\mathrm{AUG}}$ interfering with Gag oligomers required to assemble complete VLPs, as the aberrant Gag-complexes do not sediment as far into the gradient. However, the binding dynamics between $18 \mathrm{~m}_{\mathrm{AUG} 1}$ and VLP assembly intermediates are likely complex and influenced by the relative amounts of the restriction factor and Gag, and the fact that $\mathrm{p} 18 \mathrm{~m}$ and Ty1 are co-expressed in the absence of pre-existing Tyl gene products.

p18m restriction of a CNC-resistant Tyl element. Since p18m $\mathrm{m}_{\mathrm{AUG} 1}$ fractionates with VLPs, is identical to the Gag CA$\mathrm{CTD}$, and the $\mathrm{CNC}^{\mathrm{R}}$ mutant Gag-A273V ${ }^{28}$ is within the critical p18m Dimer-1 interface, p18m CNC might be mediated by a p18m-Gag interaction within this region. However, the relative level of Gag and p22/p18 and timing of expression influence restriction, as evidenced by loss of CNC-resistance when wild type p22 is inducibly co-expressed at a higher level than a Ty1 Gag-CNC $^{\mathrm{R}}$ mutant $^{28,53}$. Here, we showed that $\mathrm{p} 18 \mathrm{~m}_{\mathrm{AUG1}}-\mathrm{A} 273 \mathrm{~V}$ restricted Ty1 mobility as well as wild-type p18m $\mathrm{m}_{\mathrm{AUG} 1}$ (Fig. $4 \mathrm{~d}-\mathrm{f}$ ), raising the possibility that inducible co-expression of Ty1 and p18m $\mathrm{m}_{\mathrm{AUG} 1}-\mathrm{A} 273 \mathrm{~V}$ may mask the effect of a heterotypic interaction. In support of this notion, uncoupling Tyl and restriction factor expression has been utilized to identify a Gag/p22 ratio optimal for isolating $\mathrm{CNC}^{\mathrm{R}}$ mutants such as $\mathrm{Gag}-\mathrm{A} 273 \mathrm{~V}^{28}$.

Therefore, we uncoupled Ty1 and $18 \mathrm{~m}_{\mathrm{AUG}}$ expression to explore protein interactions genetically. Isogenic strains containing an endogenously expressed chromosomal insertion of a WT or A273V Ty1his3-AI element were analysed for Ty1 mobility following inducible expression of $\mathrm{p} 18 \mathrm{~m}_{\mathrm{AUG}}, \mathrm{p} 18 \mathrm{~m}_{\mathrm{AUG1}}-\mathrm{A} 273 \mathrm{~V}$ or empty vector. Restriction of WT and A273V Ty1 elements by p $18 \mathrm{~m}_{\mathrm{AUG1}}-\mathrm{WT}$ and A273V were compared in a pairwise fashion, normalized to Tyl mobilities in the absence of the restriction factor, and strains were verified for comparable levels of Gag$\mathrm{A} 273 \mathrm{~V}$ and $\mathrm{p} 18 \mathrm{~m}_{\mathrm{AUG} 1}-\mathrm{A} 273 \mathrm{~V}$ relative to WT (Fig. 6g, Supplementary Fig. 7 and Supplementary Table 1). WT-WT or A273VA273V restricted significantly better than heterotypic pairings of $\mathrm{p} 18 \mathrm{~m}_{\mathrm{AUG} 1}$ and Ty1. These results support a model of $\mathrm{p} 18 \mathrm{~m}_{\mathrm{AUG}}$ restriction in which Dimer-1 residues interact with the corresponding residues in Gag and interfere with proper Gag function. The heterotypic pairing of Gag-A273V with $\mathrm{p} 18 \mathrm{~m}_{\mathrm{AUG} 1}-\mathrm{A} 273$ may also contribute to CNC-resistance.

\section{Discussion}

Genetic dissection of Tyl initially showed that a C-terminal domain in Gag (UBN2) is contained within the retrotransposon restriction factor $\mathrm{p} 22 / \mathrm{p} 18^{28}$ (Fig. 1a). Our crystallographic and biophysical analyses of bacterially expressed p18m derived from this region greatly extend these genetic studies by revealing that UBN2-p22 is highly related to the Gag CA-CTD of several retroelements or exapted Gag proteins ${ }^{54-56}$. DALI comparisons reveal weaker but significant matches to CA proteins of infectious retroviruses such as MLV and HIV-1 as well as the human endogenous retrovirus HERV-K (Supplementary Fig. 1). Our findings expand the view that the CA gene of LTR retrotransposons share a common evolutionary origin ${ }^{41,42,57}$.

Biophysical studies of $\mathrm{p} 18 \mathrm{~m}$ reveal a stable obligate dimer with a tendency to form higher-order structures, and crystal studies indicate the presence of two possible dimer interfaces in $\mathrm{p} 18 \mathrm{~m}$ (Fig. 2). Dimer-1 involves a largely hydrophobic interaction between the exposed surfaces of $\alpha 1$ and $\alpha 3$ on two opposing monomers whereas the Dimer- 2 interaction is through the side chains of residues exposed on the exterior of $a 4$ and $a 5$. The importance of Dimer- 1 for $\mathrm{p} 18 \mathrm{~m}$ integrity and function is evident from mutational analyses in bacteria and yeast as introduction of changes likely disruptive to Dimer-1 results in loss of protein integrity and only conservative changes are tolerated (Supplementary Table 4). The core Dimer-1 interface residues I269 and 
a

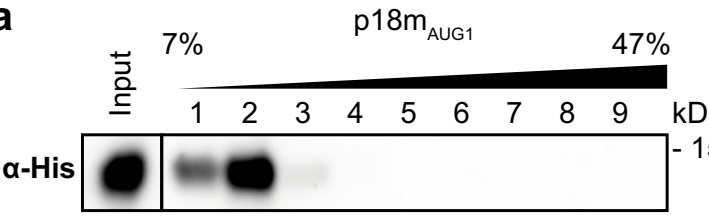

Longer
exposure

b

b

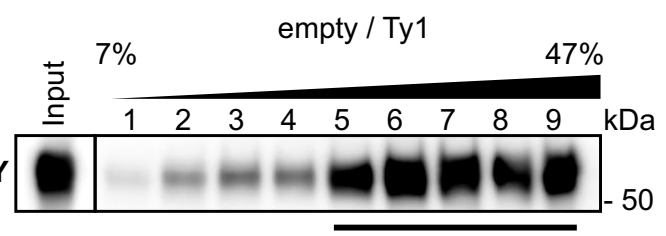

C

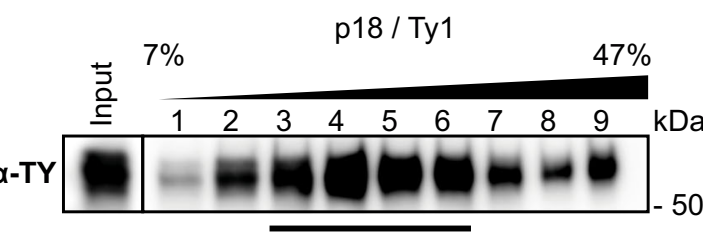

d

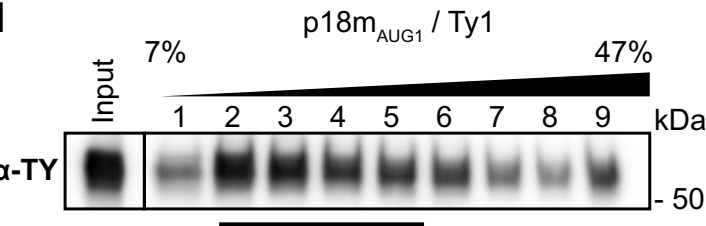

a-His

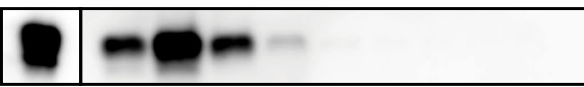

Longer exposure

e
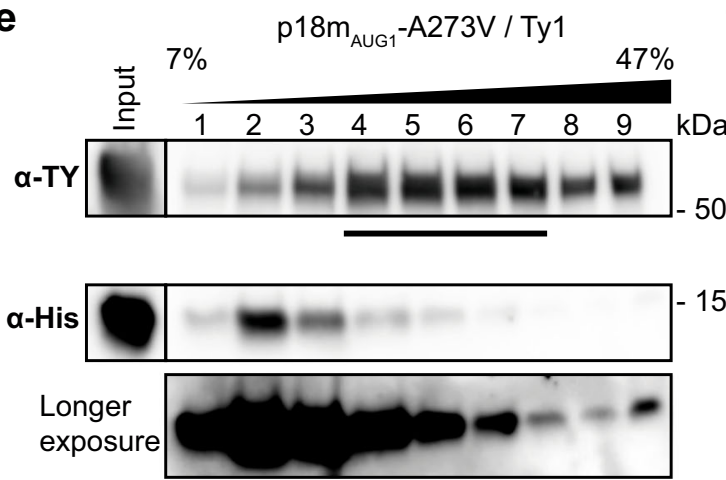

-15 a-His

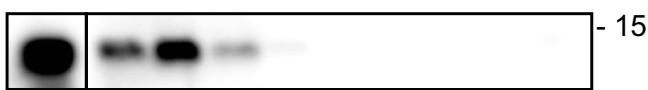

Longer exposure

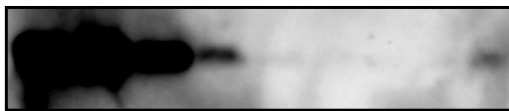

$\mathbf{f}$

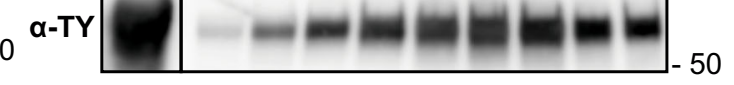

g
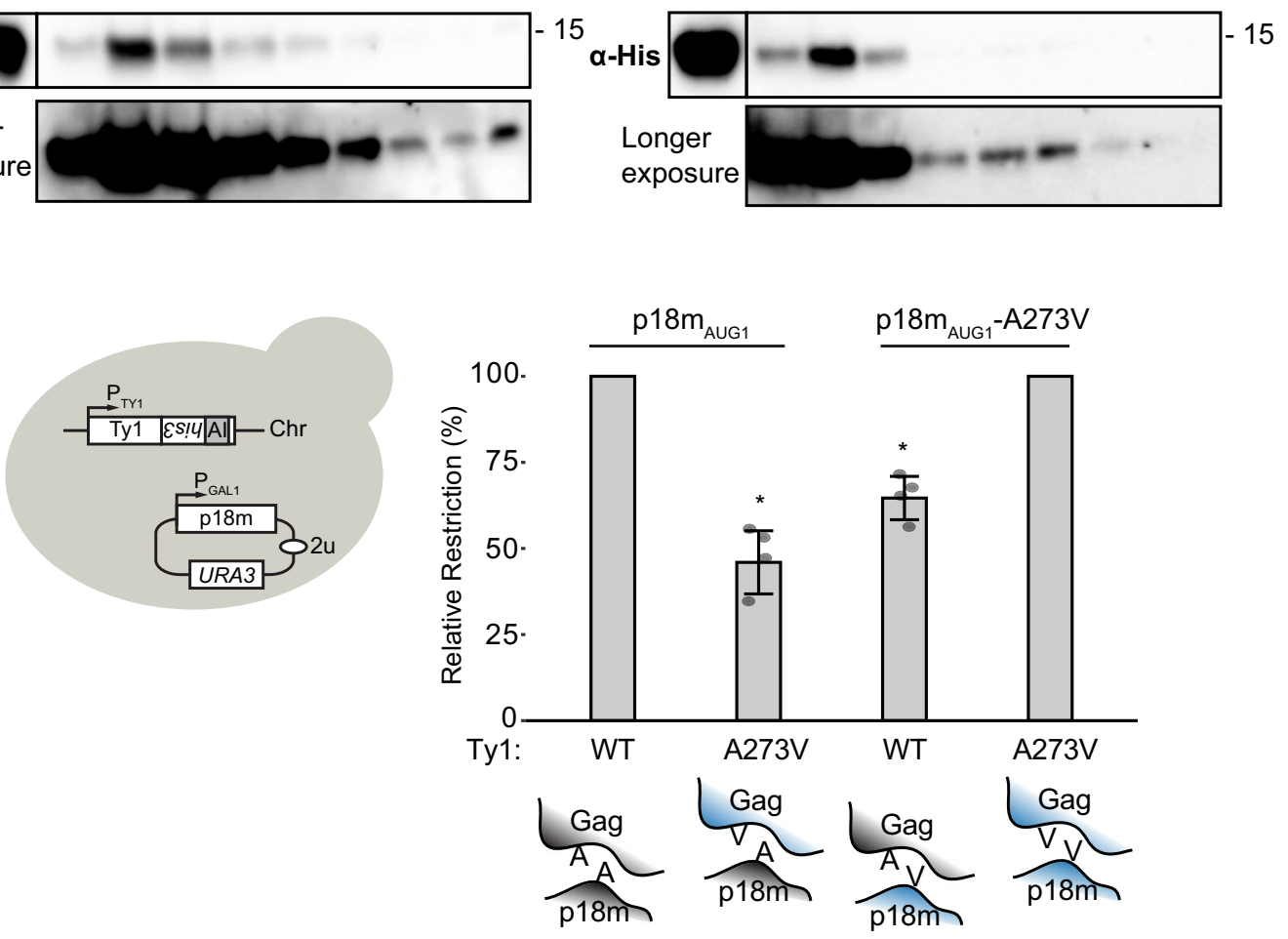

A273 are generally more sensitive to disruption both in the context of Gag and p18m. However, whilst Gag is more tolerant to polar substitutions at residues V270 and L312, just peripheral to the core, the L312S mutation is not tolerated in the context of $\mathrm{p} 18 \mathrm{~m}$.
NNK mutagenesis revealed exceptional substitutions in the Dimer-1 core interface as $\mathrm{p} 18 \mathrm{~m}_{\mathrm{AUG} 1}$ tolerates A273S and A273C. Bacterially expressed $\mathrm{p} 18 \mathrm{~m}_{\mathrm{AUG} 2}-\mathrm{A} 273 \mathrm{C}$ still forms strong dimers under reducing conditions when assessed by MALLS (Supplementary Fig. S8a) and mass spectrometry analysis demonstrates 
Fig. 6 p18m-Gag interaction is critical for restriction activity. a-f Protein extracts from galactose-induced yeast cells (Input) were fractionated over a 7-47\% continuous sucrose gradient and immunoblotted. The bars below anti-TY blots denote peak Gag fractions containing more than 1/9 of the Gag signal across the gradient, as determined by densitometric analysis. A representative image of at least 3 replicates is shown. Strains used: DG4292 (a), DG3739 (b), DG4162 (c), DG4147 (d), DG4165 (e), DG4340 (f). Migration of molecular weight standards is shown alongside the immunoblots. Images of the whole gel immunoblots are provided in the Source Data file. $\mathbf{g}$ (Left) Schematic illustrating endogenously expressed, chromosomal Ty1 and galactoseinducible, plasmid-borne $18 \mathrm{~m}_{\mathrm{AUG}}$. Ty1 is tagged with the his3-Al retromobility indicator gene; histidine prototrophy requires retromobility. (Right) Relative restriction by $\mathrm{p} 18 \mathrm{~m}_{\mathrm{AUG}}$ and $\mathrm{p} 18 \mathrm{~m}_{\mathrm{AUG} 1}-\mathrm{A} 273 \mathrm{~V}$ of Ty1 and Ty1-A273V for homotypic and heterotypic pairings. The relative restriction is calculated as the percentage of fold-restriction by the homotypic pairing. Quantitative mobility data is mean from four replicates of galactose-induced cells. Each bar represents the mean of the four independent measurements displayed as points. The error bar centre represents the mean of the four measurements and the error bar extent \pm the standard deviation. Significance is calculated from a two-sided Student's $t$-test compared with homotypic relative restriction $\left({ }^{\star} p<0.05\right.$, exact $p$-values provided in Supplementary Table 1). The cartoons below illustrate homotypic or heterotypic p18m-Gag interactions. See also Supplementary Table 1 and Supplementary Fig. 7. Source data is provided in the Source Data file.

there are no covalently linked chains (Supplementary Fig. S8b). While the rules governing the Dimer-1 interface remain incomplete, our data imply that Gag likely has more binding interfaces to stabilize the protein than does the p18m fragment, which appears to form obligate dimers relying on the hydrophobic Dimer-1 interface to retain restriction factor activity.

An essential step in the replication of retroviruses and LTR retrotransposons involves the assembly of Gag into a shell surrounding their RNA genomes. These fullerene structures are made up of arrays of hexameric and pentameric Gag or CA. A number of studies employing cryo-electron tomographic and single-particle analysis of native viral particles or in vitro assemblies have been performed to study these shell structures ${ }^{32-37}$ and to characterise the interactions involving the NTDs and CTDs of CA. These include the NTD-NTD interactions that build the hexamer or pentamer, the CTD-CTD interactions that link the neighbouring capsomeres and NTD-CTD interactions that stabilize the overall structure. Importantly, the architecture of the CTD-CTD interaction is highly conserved. Inspection of the $18 \mathrm{~m}$ Dimer-1 interface from Ty1 reveals that it is highly related to dimer interfaces in $\mathrm{Ty} 3^{41}$ and the dARC proteins from Drosophila ${ }^{42,43}$, and more distantly to retroviral structures (Supplementary Fig. 2). Given these CA-CTD dimer structures all contain the same hydrophobic core and surrounding salt bridges, this implies a similar role for Dimer-1 in Tyl VLP assembly.

The importance of the Dimer-2 interface for p18m structure and Gag function is revealed by biophysical and genetic analyses, and molecular modelling. The p18m-F323S substitution within a4 suppresses higher-order oligomers but does not affect Dimer-1 interaction (Fig. 3d), raising the possibility of another function for Dimer-2. Previous genetic analyses show that an in-frame codon insertion at Gag-I341 affects transposition, amino acid substitution Gag-I343K within a5 alters VLP assembly ${ }^{58-60}$, and GagV336I within a5 confers weak CNC-resistance ${ }^{28}$. Here, we characterise F323 substitutions of Dimer-2 in the context of $\mathrm{p} 18 \mathrm{~m}_{\mathrm{AUG} 1}$ and Gag. Remarkably, a separation of function phenotype is observed for F323S and F323D. Neither substitution affects restriction (Fig. 3e, f), but both greatly decrease Tyl mobility when placed in GAG (Fig. $3 \mathrm{~g}, \mathrm{~h}$ ). The data suggest that the Dimer-2 interface may maintain the Ty1 Gag particle 3-fold axis (Supplementary Figure 4) that is required for normal VLP assembly and transposition but is not required for $\mathrm{p} 18 \mathrm{~m}_{\mathrm{AUG} 1}$ restriction.

Given that $18 \mathrm{~m}$ is identical to the CA-CTD of Tyl Gag, we propose a structural model for $\mathrm{CNC}$, where the insertion of $\mathrm{p} 22 /$ p18 into the VLP lattice during assembly produces nonproductive or dead-end interactions (Fig. 7). In the normal course of VLP formation, Ty1 Gag assembles into complete VLPs through NTD-NTD, NTD-CTD, and CTD-CTD interactions that are all required to form a closed intact shell. If $\mathrm{p} 18 \mathrm{~m}$ is introduced via a homotypic interaction with the CTD of Gag, as suggested by pairwise interactions of p18m and Gag (Fig. $6 \mathrm{~g}$ ), normal assembly is unable to further propagate because it lacks an NTD. This will result in partial VLP and/or incomplete lattice structures. The dispersion of VLP assemblies following sucrose gradient sedimentation also supports this model (Fig. 6a-f). Since the state of capsid assembly is essential for productive reverse transcription in both the spuma- and ortho-retroviruses ${ }^{61-63}$, the specific architecture of Ty1 VLPs should also affect critical steps in the Ty1 replication cycle. Indeed, several defects in the process of Ty1 retrotransposition are detected during $\mathrm{CNC}^{24,53}$. Reverse transcription intermediates and full-length cDNA are not detected, PR cleaves Pol proteins aberrantly, mature IN fails to accumulate, and Ty1 RNA is more sensitive to ribonuclease treatment $25,27,28,64,65$. We hypothesize that some limited incorporation of $\mathrm{p} 22$ is tolerated, allowing PR-mediated conversion of p22 to p18. However, once a threshold level of p22/p18 is reached, it would become impossible to build the stable VLP shells required for protein maturation, reverse transcription, and integration due to Gag oligomers becoming poisoned by the incorporation of $\mathrm{p} 18$.

Analysis of the Gag-A273V $\mathrm{CNC}^{\mathrm{R}}$ mutation also supports the idea that a homotypic interaction at the Dimer-1 interface enhances VLP formation. In the context of p18m, A273V subtly alters the p18m Dimer-1 interface (Fig. 5) and in the context of Gag, A273V does not affect transposition (Fig. 4). However, GagA273V confers resistance to p22 and attenuates PR-mediated processing of $\mathrm{p} 22-\mathrm{p} 18^{28}$, suggesting that structural changes affect access to $\mathrm{PR}$ and $\mathrm{CNC}^{\mathrm{R}}$ in vivo. Here, we provide evidence that heterotypic pairings of A273 and A273V on p18m and Gag promote $\mathrm{CNC}^{\mathrm{R}}$ when compared with homotypic pairings (Fig. 6g). Therefore, $\mathrm{CNC}^{\mathrm{R}}$ may result from the reduced association at the Dimer-1 interface between heterotypic p18m and Gag-A273V during VLP assembly.

Data from the two yeast co-expression systems utilized here support the idea that p18m interferes at an early VLP assembly step and raises additional questions concerning the resistance of preformed Gag-complexes to p18m. In a Tyl-less strain background, ectopic overexpression of $\mathrm{p} 18 \mathrm{~m}$ and Ty1 from galactose-inducible promoters results in potent restriction (Figs. 1 and 4 and Supplementary Table 1$)^{25,28}$. However, the same galactose-induced p $18 \mathrm{~m}$ against endogenously expressed Tyl results in much lower restriction (Fig. 6G and Supplementary Table 1). Constitutive native expression of a chromosomal Tyl element may allow threshold steps in VLP assembly to begin prior to induction of $\mathrm{p} 18 \mathrm{~m}$. The strong resistance that early expression confers on Tyl, independent of any mutations, implies an important but incompletely defined kinetic component to CNC, and suggests p22 acts at an early VLP assembly step, consistent with our model.

Importantly, our model explains the negative feedback loop proposed for $\mathrm{CNC}^{24-26}$. As the Ty1 copy number increases, the 


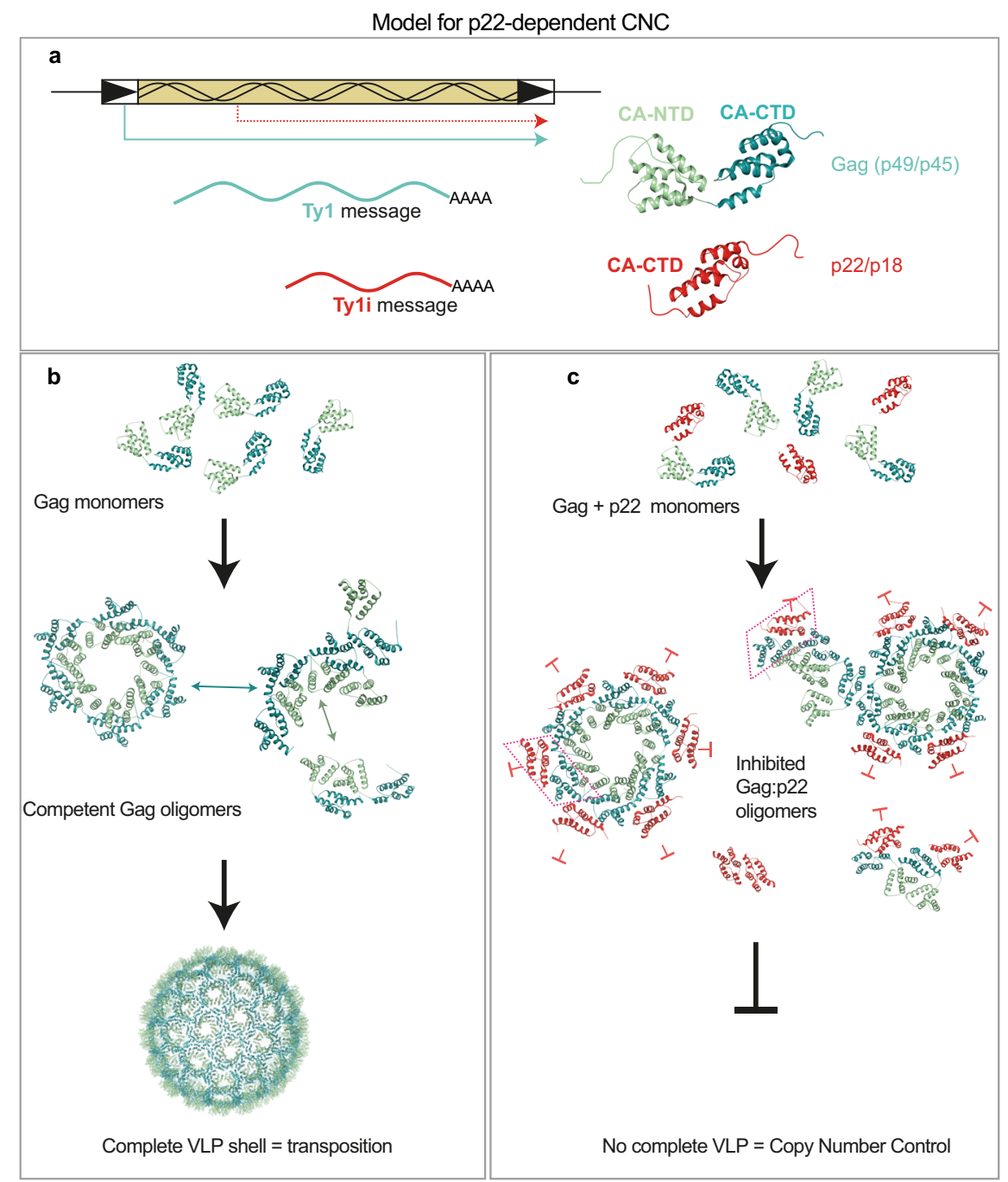

Fig. 7 Model for p22/p18-dependent CNC. a Transcription of Ty1 results in either a full-length Ty1 transcript (teal), or a short, inhibitory transcript Ty1i (red). The long transcript encodes full-length Ty1 Gag and Pol proteins, shown in green/cyan cartoon. The shorter Ty1i transcript encodes p22/p18, a C-terminal portion of Gag equivalent to a CA-protein CTD, lacking an NTD (red cartoon), as well as Pol proteins, which are not required for restriction ${ }^{25}$. b Full-length Gag monomers assemble into oligomers through NTD-NTD, and CTD-CTD interactions to construct an icosahedral shell, constituting the VLP, which is required for transposition. $\mathbf{c}$ In the presence of p22/p18, Gag monomers are also able to oligomerise with p22/p18 through CTD-CTD interactions. Two examples of a Gagp22/p18 hetero-interaction through the CTD interface are highlighted by the trapezoidal boxes. While Gag proteins may be able to homo-oligomerise through their NTDs, Gag oligomers that associate with p22/p18 become assembly dead ends resulting in partially assembled particles that cannot support transposition.

expression of $\mathrm{p} 22$, and therefore sequestration of Gag, also increases. At a certain Ty1 copy number, the p22 threshold level is reached, thus stabilising the number of genomic elements, and preventing runaway transposition from compromising the host genome. In the reference strain S288C, this appears to be $\sim 32 \mathrm{Ty} 1$ elements. Nevertheless, differing affinities of the CTD-CTD interface, expression of p22, or horizontal transfer of novel GAG sequences from other species might modulate this threshold level in related elements or different strains, resulting in differing genomic copy numbers to reach the threshold level of $\mathrm{p} 22^{6}$.

Ty1 CNC mediated by p22 is related to other Gag-like restriction factors active against retroviruses. Precedence for restriction through the interference of assembly comes from endogenous HERV-K inhibition of HIV-1 particle assembly66,67. In addition, ovine restriction factors derived from endogenous Jaagsiekte retroviruses are altered Gag proteins that block viral Gag trafficking during a late stage of oncogenic Jaagsiekte retroviral infection ${ }^{68,69}$. Gag-like restriction factors also act at the post-entry phase of retroviral infection. Fv1 is an exapted restriction factor encoded by an endogenous retroelement $G A G$ gene that has been active in the Muroidea superfamily of mammals for at least 45 million years ${ }^{70,71}$. Fvl alleles selectively restrict MLV infection at a step between reverse transcription and integration through interactions with the Gag core ${ }^{72}$. Comparative structural analyses between Fv1 and HIV restriction factors Trim5a and TrimCypA reveal how the extended antiparallel organization of the dimeric restriction factor enhances their affinity for a preformed HIV lattice ${ }^{73}$.

Our work raises the possibility that CA-CTD, CA-NTD domains, or perhaps additional coding segments derived from GAG or $P O L$ exist as exapted restriction factors active against infectious or endogenous retroelements. Genome sequence comparisons reveal S. cerevisiae strains that contain truncated Ty sequences with coding potential at high allele frequency $6,74,75$. There is also a growing body of evidence for the exaptation of 
endogenous retroviral gene segments in vertebrates ${ }^{54,76,77}$, with many of these involving $G A G$.

\section{Methods \\ Yeast strains and plasmids. Strains and plasmids are listed in Supplementary Table 5 and Supplementary Table 6, respectively. Standard yeast genetic and microbiological techniques were used in this work ${ }^{78}$. All Tyl nucleotide and amino acid information correspond to the Ty1H3 sequence (GenBank M18706.1) ${ }^{79}$. pGAL-Yes2 (pBDG1293, Invitrogen cat. no. V825-20) derived plasmids were generated by cloning custom commercial gene fragments (Integrated DNA Tech- nologies and Twist Bioscience) using XhoI and EcoRI with NEBuilder HiFi DNA Assembly Master Mix (New England Biosciences cat. no. E2621). All plasmids generated were verified by DNA sequencing.}

Yeast Media. For galactose induction in liquid media, starter cultures were grown overnight at $30^{\circ} \mathrm{C}$ in synthetic media containing $2 \%$ raffinose, diluted $1: 20$ into media containing $2 \%$ galactose, and grown at $22{ }^{\circ} \mathrm{C}$.

Ty1his3-AI mobility assays. Tyl retromobility events were detected using the his3-AI retromobility indicator gene ${ }^{44}$ by qualitative and quantitative assays ${ }^{25}$. Qualitative assays were printed from glucose plates onto galactose plates, grown for $48 \mathrm{~h}$ at $22^{\circ} \mathrm{C}$, then printed to glucose plates lacking histidine and grown at $30^{\circ} \mathrm{C}$. Quantitative retromobility frequencies were determined from quadruplicate galactose inductions diluted in water, plated on synthetic dropout media, and colonies counted. All experiments were galactose-induced for $48 \mathrm{~h}$ at $22^{\circ} \mathrm{C}$, except for strains DG4296-98 and DG4279-81 which were galactose-induced for $24 \mathrm{~h}$. Data represent at least four independent galactose inductions; $p$-values were calculated by two-sided Student's $t$-test. Complete data, including standard deviations and $p$-values, are listed in Supplementary Table 1.

Immunoblotting. Immunoblotting of total protein from galactose-induced yeast prepared by trichloroacetic acid (TCA) precipitation was performed using standard techniques ${ }^{25}$. Cells were broken by vortexing in the presence of glass beads in $20 \%$ TCA and washed in 5\% TCA. Proteins were separated on 15\% (for detecting p18 constructs), 10\% (for detecting Pgk1), or 8\% (for detecting Gag) SDS-PAGE gels. PVDF membranes were immunoblotted with antibodies at the following dilutions in $2.5 \%$ milk-TBST: polyclonal rabbit p18 antisera $(1: 5000)^{25}$, monoclonal rabbit hexa-histidine antibody clone RM146 (ThermoFisher cat. no. MA5-33032) $(1: 1000)$, mouse monoclonal anti-TY tag antibody clone BB2 $(1: 5000)^{80}$ or mouse monoclonal anti-Pgk1 antibody clone 22C5D8 (Invitrogen cat. no. 459250) (1:1000). Immune complexes were detected with WesternBright enhanced chemiluminescence (ECL) detection reagent (Advansta cat. no. K-12049-D50). All imaging was done using a ChemiDoc MP (Bio-Rad). Precision Plus Kaleidoscope protein standards (Bio-Rad cat. no. 1610395) were used to estimate molecular weights. Total protein was detected by running samples on a 10\% TGX StainFree $^{\mathrm{TM}}$ FastCast ${ }^{\mathrm{TM}}$ Acrylamide gel (Bio-Rad cat. no. 1610173) and gel-imaging after $45 \mathrm{~s}$ of activation. Protein quantification with total protein normalization was performed using Image Lab (Bio-Rad).

Protein expression and purification. The DNA sequence for S. cerevisiae Ty1A (Uniprot P08405), codon-optimised for expression in E. coli, was synthesised by GeneArt. Sequences corresponding to residues M249-N355 (p18m $\left.\mathrm{AUG1}_{1}\right)$ and M259-N355 (p18m $\mathrm{m}_{\mathrm{AUG} 2}$ ) were amplified by PCR and the products were inserted into a pET22b expression vector (Novagen) between the NdeI and Xhol restriction sites in order to produce $\mathrm{C}$-terminal fusion proteins containing the hexa-histidine tag PLEHHHHHH. Mutations were introduced into these parent constructs using the Quikchange II XL site-directed mutagenesis kit (Agilent) following the manufacturer's instructions. The codon optimised p18m DNA sequence and primer sequences for PCR and mutagenesis are provided in Supplementary Table 7.

p18m proteins were expressed in the E. coli strain BL21 (DE3) grown in LBbroth by induction of log-phase cultures with $1 \mathrm{mM}$ IPTG, followed by incubation overnight at $20^{\circ} \mathrm{C}$ with shaking. Cells were pelleted and resuspended in $50 \mathrm{mM}$ Tris- $\mathrm{HCl}, 150 \mathrm{mM} \mathrm{NaCl}, 10 \mathrm{mM}$ Imidazole, $5 \mathrm{mM} \mathrm{MgCl}, 1 \mathrm{mM}$ DTT, pH 9.0, supplemented with $1 \mathrm{mg} \mathrm{mL}^{-1}$ lysozyme (Sigma-Aldrich), $10 \mu \mathrm{gL}^{-1}$ DNase I (Sigma-Aldrich), and 1 Protease Inhibitor cocktail tablet (EDTA free, Pierce) per $40 \mathrm{~mL}$ of buffer. Cells were lysed using an EmulsiFlex-C5 homogeniser (Avestin) and His-tagged protein captured from the clarified lysate using immobilised metal ion affinity on a $5 \mathrm{~mL} \mathrm{Ni}^{2+}$-NTA Superflow column (Qiagen).

For crystallographic analysis of $\mathrm{p} 18 \mathrm{~m}_{\mathrm{AUG} 1}$ and $\mathrm{p} 18 \mathrm{~m}_{\mathrm{AUG} 2}-\mathrm{A} 273 \mathrm{~V}, \mathrm{Ni}^{2}+-\mathrm{NTA}$ bound proteins were eluted with $50 \mathrm{mM}$ Tris- $\mathrm{HCl}, 150 \mathrm{mM} \mathrm{NaCl}, 250-300 \mathrm{mM}$ Imidazole $\mathrm{pH}$ 9.0. Carboxypeptidase A (Sigma C9268) was added at 1:100 (w:w) ratio and the resulting mixture was incubated overnight at $4{ }^{\circ} \mathrm{C}$ to digest the $\mathrm{C}$-terminal his-tag. The Carboxypeptidase $\mathrm{A}$ was then inactivated by the addition of $2 \mathrm{mM}$ Tris (2-carboxyethyl) phosphine (TCEP) and proteins further purified by gel filtration chromatography on a Superdex ${ }^{\mathrm{TM}} 75(26 / 60)$ column equilibrated in $20 \mathrm{mM}$ Tris- $\mathrm{HCl}, 150 \mathrm{mM} \mathrm{NaCl}, 1 \mathrm{mM}$ TCEP $\mathrm{pH} 9.0$.

For $\mathrm{p} 18 \mathrm{~m}_{\mathrm{AUG}}$, the protein was transferred to Acetate Buffer $(50 \mathrm{mM}$ Sodium Acetate, $300 \mathrm{mM} \mathrm{NaCl}, 1 \mathrm{mM}$ TCEP pH 5.0). The, $\mathrm{Ni}^{2}{ }^{+}$-NTA eluent was first diluted 1:4 with $2 \times$ Acetate Buffer, the $\mathrm{pH}$ adjusted to 5.0 with $\mathrm{HCl}$, and then the protein dialysed exhaustively against $2 \mathrm{~L}$ of Acetate Buffer overnight (SnakeSkin dialysis tubing, $10 \mathrm{kDa}$ MWCO, ThermoFisher). $\mathrm{p} 18 \mathrm{~m}_{\mathrm{AUG} 2}$ was then further purified by gel filtration chromatography on a Superdex ${ }^{\mathrm{TM}} 75(26 / 60)$ column equilibrated in Acetate Buffer. Seleno-methionine $\mathrm{p} 18 \mathrm{~m}_{\mathrm{AUG} 2}$ was produced using an identical procedure, except E. coli B834 (DE3) cells, grown in selenomethionine Medium (Molecular Dimensions, Newmarket, UK), were used to express the protein.

Electrospray-ionisation mass spectrometry (ESI-MS) was used to determine protein molecular masses of WT and mutants, ascertain the degree of selenomethionine incorporation, and confirm His-tag removal where appropriate. Usually, complete digestion left a C-terminal PLEH remnant. Purified proteins were concentrated by centrifugal ultrafiltration (Vivaspin, MWCO $10 \mathrm{kDa}$ ), then snap-frozen and stored at $-80^{\circ} \mathrm{C}$. Protein concentrations were determined by UV absorbance spectroscopy using a calculated extinction coefficient at $280 \mathrm{~nm}$.

Protein crystallisation. $\mathrm{p} 18 \mathrm{~m}$ proteins were crystallised using sitting drop vapour diffusion at $18^{\circ} \mathrm{C}$, using Swissci MRC 2-drop trays (Molecular Dimensions) with drops set using a Mosquito robot with humidity chamber (TTP Labtech).

Initial trials using native $18 \mathrm{~m}_{\mathrm{AUG} 2}$ produced only poorly diffracting fibrous needles. However, crystals of Se-Met p18m $\mathrm{m}_{\mathrm{AUG} 2}$ were obtained using $13.6 \mathrm{mg} \mathrm{mL}^{-1}$ protein in $50 \mathrm{mM}$ Sodium Acetate $\mathrm{pH} 5.0,300 \mathrm{mM} \mathrm{NaCl} 1 \mathrm{mM}$ TCEP and mother liquor containing glycerol and PEG $4 \mathrm{~K}$. Optimisation of these conditions including microseeding (Seed Bead kit, Hampton) produced the best crystal in a condition containing a mixture of $260 \mathrm{~nL}$ protein $\left(13.6 \mathrm{mg} \mathrm{mL}^{-1}\right), 120 \mathrm{~nL}$ precipitants $(27.9 \%$ Glycerol, 17.7\% PEG $4 \mathrm{~K}$ 0.1 M HEPES pH 7.5), $20 \mathrm{~nL}$ seed solution (seeds produced in a solution of $100 \%$ mother liquor of $20 \%$ Glycerol, $31 \%$ PEG 4 K $0.1 \mathrm{M}$ HEPES $\mathrm{pH}$ 7.5). The large majority of drops with this condition produced crystals that were over-nucleated thin needles or spherulites. However, in one drop a hexagonal crystal appeared after $\sim 8$ days and reached a maximal size of $80 \times 80 \times 250 \mu \mathrm{m}$ after $\sim 21$ days before it was harvested into liquid nitrogen using a lithographic loop (MiteGen) and mother liquor as cryoprotectant.

Well-diffracting crystals of $\mathrm{p} 18 \mathrm{~m}_{\mathrm{AUG} 1}$ were obtained with $6.25 \mathrm{mg} \mathrm{mL}^{-1}$ protein in $20 \mathrm{mM}$ Tris- $\mathrm{HCl} \mathrm{pH} \mathrm{8.5,150} \mathrm{mM} \mathrm{NaCl,} 1 \mathrm{mM}$ TCEP using $200 \mathrm{~nL}$ protein solution, and $200 \mathrm{~nL}$ of mother liquor with $\mathrm{pH}$ ranging from 7.5-9.0 and containing between 1.125-1.250 $\mathrm{M} \mathrm{Li}_{2} \mathrm{SO}_{4}$. The best crystal was a hexagonal prism $\sim 160 \times 160 \times 160 \mu \mathrm{m}$ and was harvested into liquid nitrogen from a drop containing $1.16 \mathrm{M} \mathrm{Li}_{2} \mathrm{SO}_{4}, 0.1 \mathrm{M}$ Tris-HCl pH 9.0 using a cryoprotectant of $1 \mathrm{M} \mathrm{Li}_{2} \mathrm{SO}_{4}, 0.1 \mathrm{M}$ Tris-HCl, 2 M Sodium Malonate, pH 7.5. p18m-A273V crystals were obtained under similar conditions, although the quality of the crystals was consistently worse. The best diffracting crystal grew in a $400 \mathrm{~nL}$ drop containing $200 \mathrm{~nL}$ protein ( $30 \mathrm{mg} \mathrm{mL}^{-1}$ ) and $200 \mathrm{~nL}$ mother liquor (1.16 $\mathrm{M} \mathrm{Li}_{2} \mathrm{SO}_{4}, 0.1 \mathrm{M} \mathrm{MES} \mathrm{pH} \mathrm{6.59).}$ Crystals were harvested into liquid nitrogen using the same cryoprotectant as for p18m $\mathrm{m}_{\mathrm{AUG} 1}$.

Data collection and structure determination. Data from Seleno-methionine crystals of $18 \mathrm{~m}_{\mathrm{AUG} 2}$ were collected at the Diamond Light Source (DLS) tuneable beamline, I04. Grid-scanning was required to ensure that only a well-diffracting portion of the crystal was exposed to the beam and a fluorescence excitation scan was recorded to determine the best wavelengths for the collection of multiwavelength anomalous dispersion (MAD) data. A high-redundancy peak-wavelength dataset was collected using an inverse-beam strategy to maximise the preservation of anomalous signals. Inverse-beam dataset halves were processed using the Xia2 pipeline ${ }^{81}$, using DIALS ${ }^{82}$ and the integrals were scaled on rotation axis before merging using AIMLESS ${ }^{83}$, yielding a single dataset with very strong anomalous differences to $3.76 \AA$ resolution. Subsequent datasets were collected at a high-energy remote and the inflection wavelength, with lower exposure and redundancy to reduce radiation damage. These datasets were processed using the xia2 pipeline using DIALS and AIMLESS, and also contained significant anomalous differences (Supplementary Table 2).

MAD Phasing was undertaken using the SHELX suite of programs ${ }^{84}$. Solutions for phases in $\mathrm{P}_{5} 22$ gave interpretable experimental density maps. Further solvent flattening using SHELXE resulted in maps that enabled the manual building of a complete model aided by the helix-finding and baton building tools of COOT ${ }^{85}$. Initial register assignment was possible due to the density of large sidechains and the positions of Seleno-methionine residues. The model was refined in PHENIX ${ }^{86}$ using the high-resolution peak-dataset and separated Bijvoet pairs to account for the strong anomalous signal in the data.

Diffraction data from $\mathrm{p} 18 \mathrm{~m}_{\mathrm{AUG} 1}$ and $\mathrm{p} 18 \mathrm{~m}_{\mathrm{AUG} 2}-\mathrm{A} 273 \mathrm{~V}$ crystals were collected at DLS beamlines I03 and I04 respectively. Data were processed using the Xia2 pipeline using DIALS and AIMLESS. The structures were solved by molecular replacement in PHASER ${ }^{87}$ implemented in the CCP4 interface ${ }^{88}$, using the p18m $\mathrm{m}_{\mathrm{AUG} 2}$ monomer as a search model.

All datasets had the same spacegroup and cell dimensions, so a consistent $R_{\text {free }}$ test set was enforced across all refinement datasets in PHENIX and REFMAC ${ }^{89}$ TLS groups, were determined using TLSMD ${ }^{90}$ and included in the final rounds of refinement when models were near complete. Throughout refinement, the model geometry was monitored and assessed using Molprobity ${ }^{91}$ and PDB-REDO ${ }^{92}$. Details of data collection, phasing, and structure refinement statistics are presented in Supplementary Table 2. 
Structure analysis and alignments. Molecular interfaces were analysed using the EBI protein structure interface analysis service PDBePISA (https://www.ebi.ac.uk/ pdbe/pisa). The Surface hydrophobicity/hydrophilicity distribution was calculated using the Pymol script (https://pymolwiki.org/index.php/Color_h). The DALI comparison server (http://ekhidna2.biocenter.helsinki.fi/dali) was used to search for and align structural homologues from the PDB. For sequence alignments and conservation analysis, Saccharomyces spp genomes obtained from SGD (https:// www.yeastgenome.org/) were searched with tBLASTn for intact Tyl-type capsid ORFs using e-value settings determined to exclude Ty2 elements and other transposons ('-evalue 1e-90'). Obtained sequences were translated, filtered to retain only those with $\geq 90 \%$ coverage of capsid, which was oriented and aligned with MAFFT ('linsi -adjustdirection -reorder') v7.453 93 and used to form a maximumlikelihood tree with FastTree v2.1.11 ${ }^{94}$. To increase the accuracy and stringency of downstream analyses, where unknown amino acid (X residue) positions could be unequivocally inferred based on the position of the sequence within the tree, determined residues were incorporated into the sequences. In total, 125 sequences were retained and used to calculate conservation scores, each corresponding to a site's evolutionary rate, using ConSurf ${ }^{48}$, these scores being used to colour the structure according to conservation. A reduced representation of the 125-sequence alignment was obtained by the selection of exemplar sequences based on the frequency of their clades within the maximum-likelihood tree. The residue colouring in the alignment is according to Clustal $\mathrm{W}^{95}$.

SEC-MALLS. Size exclusion chromatography coupled multi-angle laser light scattering (SEC-MALLS) was used to determine the molar mass distribution of $\mathrm{p} 18 \mathrm{~m}$ and $18 \mathrm{~m}$ interface mutants. Samples ranging from $100-400 \mu \mathrm{M}$ were applied in a volume of $100 \mu \mathrm{L}$ to a Superdex ${ }^{\mathrm{TM}}$ INCREASE $20010 / 300 \mathrm{GL}$ column equilibrated in Acetate Buffer at a flow rate of $1.0 \mathrm{~mL} \mathrm{~min}{ }^{-1}$. The scattered light intensity and the protein concentration of the column eluate were recorded using a DAWN-HELEOS laser photometer and OPTILAB-rEX differential refractometer respectively. The weight-averaged molar mass of material contained in chromatographic peaks was determined from the combined data from both detectors using the ASTRA software version 7.3.2.19 (Wyatt Technology Corp., Santa Barbara, CA, USA).

Analytical ultracentrifugation. Sedimentation velocity experiments were performed in a Beckman Optima Xl-I analytical ultracentrifuge using conventional aluminum double sector centrepieces and sapphire windows. Solvent density and the protein partial specific volumes were determined as described ${ }^{96}$. Prior to centrifugation, $\mathrm{p} 18 \mathrm{~m}$ and $\mathrm{p} 18 \mathrm{~m}$ interface-mutant samples were prepared by exhaustive dialysis against the buffer blank solution (Acetate Buffer). Samples $(420 \mu \mathrm{L})$ and buffer blanks $(426 \mu \mathrm{L})$ were loaded into the cells and centrifugation was performed at 50,000 rpm $(182,000 \times g)$ and $293 \mathrm{~K}$ in an An50-Ti rotor. Interference data were acquired at time intervals of $180 \mathrm{~s}$ at varying sample concentrations $(30-181 \mu \mathrm{M})$ using the ProteomeLab 6.04 software. Data recorded from moving boundaries were analysed in terms of the continuous sedimentation coefficient distribution function $\mathrm{C}(\mathrm{S})$ using the program SEDFIT ${ }^{97}$.

Sedimentation equilibrium experiments were performed in a Beckman Optima XL-I analytical ultracentrifuge using aluminum double sector centrepieces in an An-50 Ti rotor. Prior to centrifugation, p18m and p18m interface-mutant samples were dialyzed exhaustively against the buffer blank (Acetate Buffer). Samples $(150 \mu \mathrm{L})$ and buffer blanks $(160 \mu \mathrm{L})$ were loaded into the cells and after centrifugation for $30 \mathrm{~h}$, interference data were collected at $2 \mathrm{~h}$ intervals until no further change in the profiles was observed. The rotor speed was then increased, and the procedure repeated. Data were collected at three speeds $18,000 \mathrm{rpm}(23,587$ $\times g), 21,000 \mathrm{rpm}(32,105 \times g)$ and $26,000 \mathrm{rpm}(49,213 \times g)$ on samples at different concentrations of $\mathrm{p} 18 \mathrm{~m}, \mathrm{p} 18 \mathrm{~m}(\mathrm{~A} 273 \mathrm{~V})$ and $\mathrm{p} 18 \mathrm{~m}(\mathrm{I} 269 \mathrm{~F})$. The program SEDPHAT $^{98}$ was used to initially determine weight-average molecular masses by nonlinear fitting of individual multispeed equilibrium profiles to a single-species ideal solution model. Inspection of these data revealed that the molecular mass showed significant concentration dependency and gave poor fits to a single species model. Therefore, global fitting of the data to a monomer-dimer-tetramer model incorporating the data from multiple speeds and multiple sample concentrations was applied to extract monomer-dimer $\left(K_{D}^{1,2}\right)$ and dimer-tetramer $\left(K_{D}{ }^{2-4}\right)$ equilibrium dissociation constants.

CD spectroscopy. Far UV CD spectra $(260-190 \mathrm{~nm})$ were recorded using a Jasco J-815 spectropolarimeter purged with nitrogen gas and equipped with a Peltier temperature controller. Spectra ( 25 accumulations) were recorded at $10^{\circ} \mathrm{C}$ in $0.1 \mathrm{~cm}$ cells at a protein concentration of $150 \mu \mathrm{g} \mathrm{mL}^{-1}$ in $10 \mathrm{mM} \mathrm{Na}$ Acetate $\mathrm{pH}$ 5.0. All spectra were corrected by subtraction of the appropriate buffer blank.

The melting profile of proteins was monitored by recording the $\mathrm{CD}$ at $222 \mathrm{~nm}$ whilst heating samples at a constant rate of $1{ }^{\circ} \mathrm{C}$ per minute from $10^{\circ} \mathrm{C}$ to $80^{\circ} \mathrm{C}$. The melting data were fitted with a spline function and the $\mathrm{T}_{\mathrm{m}}$ for thermal transitions determined from the maximum of the 1st derivative.

NNK mutant screen. Gene fragment libraries (Integrated DNA Technologies) containing a randomized NNK codon $(\mathrm{N}=\mathrm{A} / \mathrm{C} / \mathrm{T} / \mathrm{G}, \mathrm{K}=\mathrm{G} / \mathrm{T})$ at either Gag-269 or Gag-273 were cloned into a pGTy1his3-AI/2 $\mu$-URA3 plasmid [pGTy1mhis3-AI (pBDG598) $]^{44}$. The vector was digested with BbvCI and BstEII, gel purified, and assembled with three overlapping gene fragments using with NEBuilder HiFi DNA Assembly Master Mix (New England Biosciences cat. no. E2621): Ty1 $1_{\text {nt.738-989, }}$

Ty $1_{\text {nt.969-1361-NNK, Ty }} 1_{\text {nt.1341-1828. }}$. The NNK plasmid library was introduced into electrocompetent TOP10 E. coli cells (Invitrogen cat. no. C404050). Approximately 1000 bacterial colonies were pooled, the plasmid DNA was extracted by midi-prep (Qiagen cat. no. 12143), then transformed into a Ty1-less yeast strain (DG3582). Yeast transformants were replica plated onto galactose plates to induce Tyl expression and grown for 2 days at $22^{\circ} \mathrm{C}$, then replica plated onto media lacking histidine, and the level of $\mathrm{His}^{+}$papillation was assessed after 3 days at $30^{\circ} \mathrm{C}$. Mutations were identified by DNA sequencing PCR-amplified regions bracketing the 269 or 273 NNK codon (primer set: 5'-GCCACAATCACAGTTTCCGC-3' and $5^{\prime}$-TGCTGTGATATCTACTGCAGCC- $3^{\prime}$ ). Select mutations were validated by recovering plasmid from yeast and sub-cloning a PCR product (primer set: $5^{\prime}$-GGTAATACATTTACTGATTCATCCTCAGC- $3^{\prime}$ and $5^{\prime}$-CCTGGAAGTGAAATTGTAGG-3') into HpaI/BstEII digested pBDG1534 (pGTy1his3-AI/TRP1 Cen) with NEBuilder HiFi DNA Assembly Master Mix (New England Biosciences cat. no. E2621). The plasmid was recovered from yeast after breaking cells by vortexing in the presence of glass beads in TNSTE-PCI (1\% Triton X-100, $0.5 \%$ SDS, $50 \mathrm{mM} \mathrm{NaCl}, 5 \mathrm{mM}$ Tris $\mathrm{pH}$ 8.0, $0.5 \mathrm{mM}$ EDTA, 50\% phenol:chloroform:isoamyl alcohol 25:24:1), then ethanol precipitating the aqueous phase and resuspending in TE. Subcloned mutant plasmids were fully sequenced to verify no secondary mutations accumulated during mutagenesis.

Sucrose gradient sedimentation. Following $48 \mathrm{~h}$ galactose induction, a $100 \mathrm{~mL}$ culture was harvested and cells were broken in $15 \mathrm{mM} \mathrm{KCl}, 10 \mathrm{mM}$ HEPES- $\mathrm{KOH}$ $\mathrm{pH} 7,5 \mathrm{mM}$ EDTA containing RNase inhibitor $(100 \mathrm{U} / \mathrm{mL})$, and protease inhibitors $\left(16 \mu \mathrm{g} \mathrm{ml}^{-1}\right.$ aprotinin, leupeptin, pepstatin A and $\left.2 \mathrm{mM} \mathrm{PMSF}\right)$ in the presence of glass beads. Cell debris was removed by centrifuging the broken cells at $10,000 \times \mathrm{g}$ for $10 \mathrm{~min}$ at $4{ }^{\circ} \mathrm{C}$. Approximately five milligrams total protein in $500 \mu \mathrm{L}$ of buffer was applied to a $7-47 \%$ continuous sucrose gradient and centrifuged using an SW41 Ti rotor at $25,000 \mathrm{rpm}(77,000 \times \mathrm{g})$ for $3 \mathrm{~h}$ at $4{ }^{\circ} \mathrm{C}$. After centrifugation, $9 \times 1.2 \mathrm{~mL}$ fractions were collected and normalized volumes of input and fractions were immunoblotted with TY-tag antibody to detect Gag and hexahistidine antibody to detect $\mathrm{p} 18 \mathrm{~m}_{\mathrm{AUG}}{ }^{28}$. Densitometric analysis was performed using Image Lab (Bio-Rad).

Reporting summary. Further information on research design is available in the Nature Research Reporting Summary linked to this article.

\section{Data availability}

For sequence conservation analysis, Saccharomyces spp genomes were obtained from SGD (https://www.yeastgenome.org/). Protein structures used in structural alignments were obtained from the Protein Data Bank (https://www.rcsb.org/). The atomic coordinates and structure factors for $\mathrm{p} 18 \mathrm{~m}_{\mathrm{AUG}}, \mathrm{p} 18 \mathrm{~m}_{\mathrm{AUG} 2}$, and $\mathrm{p} 18 \mathrm{~m}_{\mathrm{AUG} 2}-\mathrm{A} 273 \mathrm{~V}$ have been deposited in the Protein Data Bank under accession numbers 7NLH [https:// doi.org/10.2210/pdb7NLH/pdb], 7NLI [https://doi.org/10.2210/pdb7NLI/pdb] and $7 \mathrm{NLG}$ [https://doi.org/10.2210/pdb7NLG/pdb]. The entire p18m sequence alignment is available to download from the Figshare repository (https://crick.figshare.com/articles/ dataset/Cottee_Supplementary_datafile1_Tylp18_alignment_txt/15060366). The Source data and whole blot images underlying Figs. 1d, 1f, 3b-c, 3f-h, 4b-c, 4e-f, 5c-d, 6a-g, and Supplementary Figs $6 \mathrm{a}-\mathrm{c}$, and 7 are provided as a Source Data file. All remaining data are contained within the article. Source data are provided with this paper.

Received: 15 March 2021; Accepted: 6 September 2021; Published online: 22 September 2021

\section{References}

1. Huang, C. R., Burns, K. H. \& Boeke, J. D. Active transposition in genomes. Annu. Rev. Genet. 46, 651-675 (2012).

2. Jern, P. \& Coffin, J. M. Effects of retroviruses on host genome function. Annu. Rev. Genet. 42, 709-732 (2008).

3. Goodier, J. L. \& Kazazian, H. H. Jr. Retrotransposons revisited: the restraint and rehabilitation of parasites. Cell 135, 23-35 (2008).

4. Lesage, P. \& Todeschini, A. L. Happy together: the life and times of Ty retrotransposons and their hosts. Cytogenetic Genome Res. 110, 70-90 (2005).

5. Curcio, M. J., Lutz, S. \& Lesage, P. The Tyl LTR-retrotransposon of budding yeast, Saccharomyces cerevisiae. Microbiol. Spectr. 3, 1-35 (2015).

6. Czaja, W., Bensasson, D., Ahn, H. W., Garfinkel, D. J. \& Bergman, C. M. Evolution of Tyl copy number control in yeast by horizontal transfer and recombination. PLoS Genet. 16, e1008632 (2020).

7. Garfinkel, D. J., Boeke, J. D. \& Fink, G. R. Ty element transposition: reverse transcriptase and virus-like particles. Cell 42, 507-517 (1985). 
8. Farabaugh, P. J. Post-transcriptional regulation of transposition by Ty retrotransposons of Saccharomyces cerevisiae. J. Biol. Chem. 270, 10361-10364 (1995).

9. Feng, Y. X., Moore, S. P., Garfinkel, D. J. \& Rein, A. The genomic RNA in Ty1 virus-like particles is dimeric. J. Virol. 74, 10819-10821 (2000).

10. Checkley, M. A., Nagashima, K., Lockett, S. J., Nyswaner, K. M. \& Garfinkel, D. J. P-body components are required for Tyl retrotransposition during assembly of retrotransposition-competent virus-like particles. Mol. Cell. Biol. 30, 382-398 (2010)

11. Malagon, F. \& Jensen, T. H. The T body, a new cytoplasmic RNA granule in Saccharomyces cerevisiae. Mol. Cell. Biol. 28, 6022-6032 (2008).

12. Sandmeyer, S. B. \& Clemens, K. A. Function of a retrotransposon nucleocapsid protein. RNA Biol. 7, 642-654 (2010).

13. Merkulov, G. V., Swiderek, K. M., Brachmann, C. B. \& Boeke, J. D. A critical proteolytic cleavage site near the $\mathrm{C}$ terminus of the yeast retrotransposon $\mathrm{Ty} 1$ Gag protein. J. Virol. 70, 5548-5556 (1996).

14. Mellor, J. et al. The Ty transposon of Saccharomyces cerevisiae determines the synthesis of at least three proteins. Nucleic Acids Res. 13, 6249-6263 (1985).

15. Cristofari, G., Ficheux, D. \& Darlix, J. L. The GAG-like protein of the yeast Tyl retrotransposon contains a nucleic acid chaperone domain analogous to retroviral nucleocapsid proteins. J. Biol. Chem. 275, 19210-19217 (2000).

16. Devine, S. E. \& Boeke, J. D. Integration of the yeast retrotransposon Ty1 is targeted to regions upstream of genes transcribed by RNA polymerase III. Gene Dev. 10, 620-633 (1996).

17. Bridier-Nahmias, A. et al. Retrotransposons. An RNA polymerase III subunit determines sites of retrotransposon integration. Science 348, 585-588 (2015).

18. Maxwell, P. H., Burhans, W. C. \& Curcio, M. J. Retrotransposition is associated with genome instability during chronological aging. Proc. Natl Acad. Sci. USA 108, 20376-20381 (2011).

19. Patterson, M. N. et al. Preferential retrotransposition in aging yeast mother cells is correlated with increased genome instability. DNA repair $34,18-27$ (2015).

20. Chan, J. E. \& Kolodner, R. D. A genetic and structural study of genome rearrangements mediated by high copy repeat Tyl elements. PLoS Genet. 7, e1002089 (2011).

21. Garfinkel, D. J. Genome evolution mediated by Ty elements in Saccharomyces. Cytogenetic Genome Res. 110, 63-69 (2005).

22. Goodier, J. L. Restricting retrotransposons: a review. Mob. DNA 7, 16 (2016).

23. Ikeda, T. et al. Intrinsic restriction activity by apolipoprotein B mRNA editing enzyme APOBEC1 against the mobility of autonomous retrotransposons. Nucleic acids Res. 39, 5538-5554 (2011).

24. Garfinkel, D. J. et al. A self-encoded capsid derivative restricts Ty1 retrotransposition in Saccharomyces. Curr. Genet. 62, 321-329 (2016).

25. Saha, A. et al. A trans-dominant form of Gag restricts Tyl retrotransposition and mediates copy number control. J. Virol. 89, 3922-3938 (2015).

26. Garfinkel, D. J., Nyswaner, K., Wang, J. \& Cho, J. Y. Post-transcriptional cosuppression of Ty1 retrotransposition. Genetics 165, 83-99 (2003).

27. Nishida, Y. et al. Ty1 retrovirus-like element Gag contains overlapping restriction factor and nucleic acid chaperone functions. Nucleic acids Res. 43, 7414-7431 (2015).

28. Tucker, J. M., Larango, M. E., Wachsmuth, L. P., Kannan, N. \& Garfinkel, D. J. The Ty1 Retrotransposon Restriction Factor p22 Targets Gag. PLoS Genet. 11, e1005571 (2015).

29. Mortuza, G. B. et al. High-resolution structure of a retroviral capsid hexameric amino-terminal domain. Nature 431, 481-485 (2004).

30. Obal, G. et al. STRUCTURAL VIROLOGY. Conformational plasticity of a native retroviral capsid revealed by x-ray crystallography. Science 349, 95-98 (2015).

31. Pornillos, O. et al. X-ray structures of the hexameric building block of the HIV capsid. Cell 137, 1282-1292 (2009)

32. Acton, O. et al. Structural basis for Fullerene geometry in a human endogenous retrovirus capsid. Nat. Commun. 10, 5822 (2019).

33. Qu, K. et al. Structure and architecture of immature and mature murine leukemia virus capsids. Proc. Natl Acad. Sci. USA 115, E11751-E11760 (2018).

34. Mattei, S., Glass, B., Hagen, W. J., Krausslich, H. G. \& Briggs, J. A. The structure and flexibility of conical HIV-1 capsids determined within intact virions. Science 354, 1434-1437 (2016).

35. $\mathrm{Ni}, \mathrm{T}$. et al. Intrinsic curvature of the HIV-1 CA hexamer underlies capsid topology and interaction with cyclophilin A. Nat. Struct. Mol. Biol. 27, 855-862 (2020).

36. Schur, F. K. et al. An atomic model of HIV-1 capsid-SP1 reveals structures regulating assembly and maturation. Science 353, 506-508 (2016).

37. Obr, M. et al. Structure of the mature Rous sarcoma virus lattice reveals a role for IP6 in the formation of the capsid hexamer. Nat. Commun. 12, 3226 (2021).

38. Gamble, T. R. et al. Structure of the carboxyl-terminal dimerization domain of the HIV-1 capsid protein. Science 278, 849-853 (1997).
39. Lanman, J. et al. Identification of novel interactions in HIV-1 capsid protein assembly by high-resolution mass spectrometry. J. Mol. Biol. 325, 759-772 (2003).

40. Lanman, J. et al. Key interactions in HIV-1 maturation identified by hydrogen-deuterium exchange. Nat. Struct. Mol. Biol. 11, 676-677 (2004).

41. Dodonova, S. O., Prinz, S., Bilanchone, V., Sandmeyer, S. \& Briggs, J. A. G. Structure of the Ty3/Gypsy retrotransposon capsid and the evolution of retroviruses. Proc. Natl Acad. Sci. USA 116, 10048-10057 (2019).

42. Cottee, M. A., Letham, S. C., Young, G. R., Stoye, J. P. \& Taylor, I. A. Structure of Drosophila melanogaster ARC1 reveals a repurposed molecule with characteristics of retroviral Gag. Sci. Adv. 6, eaay6354 (2020).

43. Erlendsson, S. et al. Structures of virus-like capsids formed by the Drosophila neuronal Arc proteins. Nat. Neurosci. 23, 172-175 (2020).

44. Curcio, M. J. \& Garfinkel, D. J. Single-step selection for Ty1 element retrotransposition. Proc. Natl Acad. Sci. USA 88, 936-940 (1991).

45. Blaszczyk, L., Biesiada, M., Saha, A., Garfinkel, D. J. \& Purzycka, K. J. Structure of Tyl Internally Initiated RNA Influences Restriction Factor Expression. Viruses 9, 74 (2017).

46. Holm, L. \& Sander, C. Dali/FSSP classification of three-dimensional protein folds. Nucleic acids Res. 25, 231-234 (1997).

47. Taylor, W. R., Stoye, J. P. \& Taylor, I. A. A comparative analysis of the foamy and ortho virus capsid structures reveals an ancient domain duplication. BMC Struct. Biol. 17, 3 (2017).

48. Ashkenazy, H. et al. ConSurf 2016: an improved methodology to estimate and visualize evolutionary conservation in macromolecules. Nucleic acids Res. 44, W344-W350 (2016).

49. Landau, M. et al. ConSurf 2005: the projection of evolutionary conservation scores of residues on protein structures. Nucleic acids Res. 33, W299-W302 (2005).

50. AL-K, H. A. et al. Yeast Ty retrotransposons assemble into virus-like particles whose T-numbers depend on the C-terminal length of the capsid protein. J. Mol. Biol. 292, 65-73 (1999).

51. Nov, Y. When second best is good enough: another probabilistic look at saturation mutagenesis. Appl Environ. Microbiol 78, 258-262 (2012).

52. Krissinel, E. \& Henrick, K. Inference of macromolecular assemblies from crystalline state. J. Mol. Biol. 372, 774-797 (2007).

53. Tucker, J. M. \& Garfinkel, D. J. Tyl escapes restriction by the self-encoded factor p22 through mutations in capsid. Mob. Genet Elem. 6, e1154639 (2016).

54. Campillos, M., Doerks, T., Shah, P. K. \& Bork, P. Computational characterization of multiple Gag-like human proteins. Trends Genet.: TIG 22 585-589 (2006).

55. Ashley, J. et al. Retrovirus-like Gag Protein Arcl Binds RNA and traffics across Synaptic Boutons. Cell 172, 262-274 e211 (2018).

56. Pastuzyn, E. D. et al. The neuronal gene arc encodes a repurposed retrotransposon gag protein that mediates intercellular RNA transfer. Cell 172, 275-288 e218 (2018).

57. Krupovic, M. \& Koonin, E. V. Homologous capsid proteins testify to the common ancestry of retroviruses, caulimoviruses, pseudoviruses, and metaviruses. J. virol. 91, e00210-17 (2017).

58. Martin-Rendon, E. et al. Structural determinants within the subunit protein of Ty1 virus-like particles. Mol. Microbiol. 22, 667-679 (1996).

59. Monokian, G. M., Braiterman, L. T. \& Boeke, J. D. In-frame linker insertion mutagenesis of yeast transposon Ty1: mutations, transposition and dominance. Gene 139, 9-18 (1994).

60. Braiterman, L. T. et al. In-frame linker insertion mutagenesis of yeast transposon Ty1: phenotypic analysis. Gene 139, 19-26 (1994).

61. Ball, N. J. et al. Structure of a spumaretrovirus Gag central domain reveals an ancient retroviral Capsid. PLoS Pathog. 12, e1005981 (2016).

62. Forshey, B. M., von Schwedler, U., Sundquist, W. I. \& Aiken, C. Formation of a human immunodeficiency virus type 1 core of optimal stability is crucial for viral replication. J. Virol. 76, 5667-5677 (2002).

63. Burdick, R. C. \& Pathak, V. K. Efficient HIV-1 in vitro reverse transcription: optimal capsid stability is required. Signal Transduct. Target Ther. 6, 13 (2021).

64. Matsuda, E. \& Garfinkel, D. J. Posttranslational interference of Tyl retrotransposition by antisense RNAs. Proc. Natl Acad. Sci. USA 106, 15657-15662 (2009).

65. Purzycka, K. J. et al. Exploring Ty1 retrotransposon RNA structure within virus-like particles. Nucleic acids Res. 41, 463-473 (2013).

66. Monde, K. et al. Molecular mechanisms by which HERV-K Gag interferes with HIV-1 Gag assembly and particle infectivity. Retrovirology 14, 27 (2017).

67. Monde, K., Contreras-Galindo, R., Kaplan, M. H., Markovitz, D. M. \& Ono, A Human endogenous retrovirus K Gag coassembles with HIV-1 Gag and reduces the release efficiency and infectivity of HIV-1. J. Virol. 86, 11194-11208 (2012).

68. Arnaud, F., Murcia, P. R. \& Palmarini, M. Mechanisms of late restriction induced by an endogenous retrovirus. J. Virol. 81, 11441-11451 (2007). 
69. Murcia, P. R., Arnaud, F. \& Palmarini, M. The transdominant endogenous retrovirus enJS56A1 associates with and blocks intracellular trafficking of Jaagsiekte sheep retrovirus Gag. J. Virol. 81, 1762-1772 (2007).

70. Boso, G., Buckler-White, A. \& Kozak, C. A. Ancient evolutionary origin and positive selection of the retroviral restriction factor Fv1 in muroid rodents. $J$. virol. 92, e00850-18 (2018).

71. Young, G. R., Yap, M. W., Michaux, J. R., Steppan, S. J. \& Stoye, J. P. Evolutionary journey of the retroviral restriction gene Fv1. Proc. Natl Acad. Sci. USA 115, 10130-10135 (2018).

72. Sanz-Ramos, M. \& Stoye, J. P. Capsid-binding retrovirus restriction factors: discovery, restriction specificity and implications for the development of novel therapeutics. J. Gen. Virol. 94, 2587-2598 (2013).

73. Goldstone, D. C. et al. Structural studies of postentry restriction factors reveal antiparallel dimers that enable avid binding to the HIV-1 capsid lattice. Proc. Natl Acad. Sci. USA 111, 9609-9614 (2014).

74. Bleykasten-Grosshans, C., Friedrich, A. \& Schacherer, J. Genome-wide analysis of intraspecific transposon diversity in yeast. BMC genomics 14, 399 (2013).

75. Carr, M., Bensasson, D. \& Bergman, C. M. Evolutionary genomics of transposable elements in Saccharomyces cerevisiae. PLoS ONE 7, e50978 (2012).

76. Ueda, M. T. et al. Comprehensive genomic analysis reveals dynamic evolution of endogenous retroviruses that code for retroviral-like protein domains. Mob. DNA 11, 29 (2020).

77. Wang, J. \& Han, G. Z. Frequent Retroviral Gene Co-option during the Evolution of Vertebrates. Mol. Biol. Evol. 37, 3232-3242 (2020).

78. Guthrie, C. \& Fink, G. Guide to yeast genetics and molecular biology. Methods Enzymol. 194, 1-863 (1991).

79. Boeke, J. D., Eichinger, D., Castrillon, D. \& Fink, G. R. The Saccharomyces cerevisiae genome contains functional and nonfunctional copies of transposon Ty1. Mol. Cell. Biol. 8, 1432-1442 (1988).

80. Bastin, P., Bagherzadeh, Z., Matthews, K. R. \& Gull, K. A novel epitope tag system to study protein targeting and organelle biogenesis in Trypanosoma brucei. Mol. biochemical Parasitol. 77, 235-239 (1996).

81. Winter, G. xia2: an expert system for macromolecular crystallography data reduction. J. Appl. Crystallogr. 43, 186-190 (2009).

82. Winter, G. et al. DIALS: implementation and evaluation of a new integration package. Acta Crystallogr D. 74, 85-97 (2018).

83. Evans, P. R. \& Murshudov, G. N. How good are my data and what is the resolution? Acta Crystallogr. Sect. D. 69, 1204-1214 (2013).

84. Sheldrick, G. M. Experimental phasing with SHELXC/D/E: combining chain tracing with density modification. Acta Crystallogr. Sect. D. 66, 479-485 (2010).

85. Emsley, P. \& Cowtan, K. Coot: model-building tools for molecular graphics. Acta Crystallogr. Sect. D. 60, 2126-2132 (2004).

86. Zwart, P. H. et al. Automated structure solution with the PHENIX suite. Methods Mol. Biol. 426, 419-435 (2008).

87. McCoy, A. J. et al. Phaser crystallographic software. J. Appl. Crystallogr. 40 658-674 (2007).

88. Winn, M. D. et al. Overview of the CCP4 suite and current developments. Acta Crystallogr. Sect. D. 67, 235-242 (2011).

89. Murshudov, G. N. et al. REFMAC5 for the refinement of macromolecular crystal structures. Acta Crystallogr. Sect. D. 67, 355-367 (2011)

90. Painter, J. \& Merritt, E. A. Optimal description of a protein structure in terms of multiple groups undergoing TLS motion. Acta Crystallogr. Sect. D., Biol. Crystallogr. 62, 439-450 (2006)

91. Chen, V. B., Wedell, J. R., Wenger, R. K., Ulrich, E. L. \& Markley, J. L. MolProbity for the masses-of data. J. Biomol. NMR 63, 77-83 (2015)

92. Joosten, R. P. et al. PDB_REDO: automated re-refinement of X-ray structure models in the PDB. J. Appl. Crystallogr. 42, 376-384 (2009).

93. Katoh, K. \& Standley, D. M. MAFFT multiple sequence alignment software version 7: improvements in performance and usability. Mol. Biol. evolution 30, 772-780 (2013).

94. Price, M. N., Dehal, P. S. \& Arkin, A. P. FastTree 2-approximately maximumlikelihood trees for large alignments. PLoS ONE 5, e9490 (2010).

95. Larkin, M. A. et al. Clustal W and Clustal X version 2.0. Bioinformatics 23, 2947-2948 (2007).
96. Laue, T. M., Shah, B. D., Ridgeway, T. M \& Pelletier, S. L. Computer-aided interpretation of analytical sedimentation data for proteins. In: Analytical Ultracentrifugation in Biochemistry and Polymer Science (eds. Harding. S. E., Rowe, A. J \& Horton, J. C.). The Royal Society of Chemistry, Cambridge United Kingdom (1992).

97. Schuck, P. Size-distribution analysis of macromolecules by sedimentation velocity ultracentrifugation and lamm equation modeling. Biophysical J. 78, 1606-1619 (2000)

98. Vistica, J. et al. Sedimentation equilibrium analysis of protein interactions with global implicit mass conservation constraints and systematic noise decomposition. Anal. Biochem. 326, 234-256 (2004)

\section{Acknowledgements}

We gratefully acknowledge the Diamond Light Source, Didcot, UK (Grant No MX13775) and beamlines I04 and I03 for access. We also acknowledge the Crick Structural Biology and Proteomics Technology Platforms for access and expertise. We thank Adam Hannon-Hatfield for valuable discussions and comments on the manuscript. This work was supported by the Francis Crick Institute, which receives its core funding from Cancer Research UK (FC001162 and FC001178), the UK Medical Research Council (FC001162 and FC001178) and the Wellcome trust (FC001162 and FC001178) and by Wellcome Trust Investigator Awards to IAT (108014/Z/15/Z) and JPS (108012/Z/15/Z). This work was also funded by an NIH grant to DJG (R01GM124216) and an NIH Postdoctoral Fellowship to SLB (F32GM139247). For the purpose of Open Access, the author has applied a CC BY public copyright licence to any Author Accepted Manuscript version arising from this submission.

\section{Author contributions}

M.A.C., S.L.B., S.C.L., S.J.K., G.R.Y. and I.A.T. performed experiments. M.A.C., S.L.B., S.C.L., S.J.K., G.R.Y., J.P.S., D.J.G. and I.A.T. contributed to experimental design and data analysis. M.A.C., S.L.B., J.P.S., D.J.G. and I.A.T wrote the paper with contributions from S.C.L, S.J.K. and G.R.Y.

\section{Competing interests}

The authors declare no competing financial or non-financial interests.

\section{Additional information}

Supplementary information The online version contains supplementary material available at https://doi.org/10.1038/s41467-021-25849-0.

Correspondence and requests for materials should be addressed to David J. Garfinkel or Ian A. Taylor.

Peer review information Nature Communications thanks Paul Lesbats and the other, anonymous, reviewer(s) for their contribution to the peer review of this work.

Reprints and permission information is available at http://www.nature.com/reprints

Publisher's note Springer Nature remains neutral with regard to jurisdictional claims in published maps and institutional affiliations.

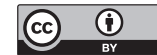

Open Access This article is licensed under a Creative Commons Attribution 4.0 International License, which permits use, sharing, adaptation, distribution and reproduction in any medium or format, as long as you give appropriate credit to the original author(s) and the source, provide a link to the Creative Commons license, and indicate if changes were made. The images or other third party material in this article are included in the article's Creative Commons license, unless indicated otherwise in a credit line to the material. If material is not included in the article's Creative Commons license and your intended use is not permitted by statutory regulation or exceeds the permitted use, you will need to obtain permission directly from the copyright holder. To view a copy of this license, visit http://creativecommons.org/ licenses/by/4.0\%

(C) The Author(s) 2021 